\title{
United Atom Force Field for Alkanes in Nanoporous Materials
}

\author{
D. Dubbeldam, ${ }^{*},{ }^{\dagger}$ S. Calero, ${ }^{\ddagger}$ T. J. H. Vlugt, ${ }^{\S}$ R. Krishna, ${ }^{\dagger}$ T. L. M. Maesen,,$"$ and B. Smit ${ }^{\dagger}$ \\ Department of Chemical Engineering, University of Amsterdam, Nieuwe Achtergracht 166, $1018 \mathrm{WV}$ \\ Amsterdam, The Netherlands, Department of Experimental Sciences, University Pablo de Olavide, \\ Ctra. Utrera km 1. 41013 Sevilla, Spain, Condensed Matter and Interfaces, Utrecht University, Utrecht \\ University, P. O. Box 80.000, 3508 TA Utrecht, The Netherlands, and ChevronTexaco, Energy Technology \\ Company, Chevron Way 100, Richmond, California 94802-0627
}

Received: December 2, 2003; In Final Form: March 22, 2004

\begin{abstract}
A novel united atom force field affords accurate and quantitative reproduction of the adsorption properties of linear and branched alkanes in nanoporous framework structures. The force field was generated by adjusting the parameters so as to faithfully reproduce the experimentally determined isotherms (particularly the inflection points) on MFI-type zeolite over a wide range of pressures and temperatures. It reproduces extremely well the Henry coefficients, heats of adsorption, preexponential factors, entropies of adsorption, and maximum loading. It is shown that the extension of the force field from MFI to other nanoporous framework topologies is successful, that it affords the prediction of topology-specific adsorption properties, and that it can be an effective tool to resolve the many discrepancies among experimental data sets.
\end{abstract}

\section{Introduction}

Molecular sieves are of importance for many refinery and petrochemical processes such as the separation of linear and branched alkanes. ${ }^{1}$ The pore sizes of these nanoporous materials are of the same order of magnitude as those of the adsorbing molecules so that adsorption can occur selectively. The performance of molecular sieves in separation and catalytic processes depends critically on the match between sieve topology and the shape and size of the adsorbate. ${ }^{2}$ It is therefore of considerable industrial importance to explore the adsorption of linear and branched alkanes in different topologies using realistic simulations at the microscopic level. ${ }^{3}$

Many molecular simulation studies have aimed at providing accurate data at a microscopic level under catalytic process conditions. ${ }^{4}$ At these conditions, adsorption properties are not readily amenable to experimental evaluation, but they are still accessible to molecular simulations. However, the simulation results are not beyond dispute, for there is no consensus on which force field is best suited to study, e.g., the adsorption of hydrocarbons in nanoporous materials. Some groups claim that an all-atom representation is required, ${ }^{5}$ whereas others assume that a united atom approach should suffice. ${ }^{6,7}$ It is also argued that three-body interactions are required for these systems. ${ }^{8}$ Within these approaches, different parameter sets have been published. Despite these differences, most studies claim a good agreement with experimental data, so that it is not trivial to select the best force field to address future practical catalytic or separation problems.

From a molecular simulation point of view, the development of a reliable force field for as wide a variety of systems as possible is of preeminent importance. Notwithstanding the

* To whom correspondence should be addressed. E-mail: D.Dubbeldam@uva.nl.

$\dagger$ University of Amsterdam.

$\doteqdot$ University Pablo de Olavide.

$\S$ Utrecht University.

"ChevronTexaco. plethora of published experimental data, these experimental results involve different zeolite samples or different experiments so that it is difficult to unambiguously compare one experiment with the next. When different experimental data are used as a calibration point to develop a molecular simulation model, the result is a different set of parameters or potentials. In this work, we develop a unique set of parameters. Although we use this approach to develop a significantly more accurate force field for hydrocarbons in nanoporous materials than previous attempts, a similar optimization strategy can be used for other systems.

The novel parameter-optimization starts by obtaining a reduced set of reliable experimental data sets, preferably of several independent research groups, to calibrate the simulations results. Next, we fit, starting with the smallest number of free parameters, and increase the number of parameters incrementally. The most important part is to analyze the physical connection between a parameter and the various adsorption properties. For example, we found that fitting to inflections in isotherms uniquely determines the adsorbate-adsorbent interaction parameters and is very sensitive to the size parameters. Inflection points in the isotherm are often related to a subtle interplay between different adsorption sites. It turns out that, if our force field can predict this interplay, it also reproduces the remaining part of the isotherm correctly. Once a reasonable set of parameters had been obtained, we reexamined the experimental data set and included those data that were consistent with the original data set. This extended data set was subsequently used to further refine the parameters. This procedure was repeated until all experimental data were accounted for. The resulting force field not only yields a superior description of the experimental data that formed the basis for the fitting procedure, but also yields an excellent description of reference systems which were not included in the calibration set.

The remainder of this paper is organized as follows. In section II, we explain the new fitting procedure. The choice of the model is discussed, followed by a screening of the experimental data 
used in the fitting procedure. The parameter optimization strategy is explained, and we present the final parameter set. This section is concluded with a detailed comparison of this work with various other models proposed in the literature. We show in section III that this procedure leads to an excellent description of adsorption properties not included in the initial optimization procedure: other sorbates, mixtures, low-coverage properties (Henry coefficients, enthalpies and entropies of adsorption), and other topologies. As an application, we have scrutinized the available experimental data indicating common sources for error. We end with some concluding remarks on the applicability of the model.

\section{Model}

A. Choice of Models and Methods. The first step in an optimization strategy is the selection of the type of force field. In the literature, one can find claims that very different force fields yield an equally good description of the adsorption isotherms. However, the following practical considerations limit the choice. The adsorption of hydrocarbons is dominated by dispersive forces. These interactions are notoriously difficult to describe using quantum chemical approaches. The most successful approach is a hybrid technique where, in addition to the ab initio quantum chemical calculation, the dispersive interactions are taken into account using ad hoc empirical potentials. $^{9}$

The next level of sophistication is to use an all-atom model. These models are commonly used in the simulations of proteins and other large systems. First attempts to simply use such a force field (consistent valence force field) for the adsorption of hydrocarbons in MFI gave a reasonable prediction of the adsorption isotherms. However, the much simpler united atom models yielded a significantly more accurate description of the adsorption isotherms..$^{5}$ Of course, this observation is not surprising since the united atom models have been specifically optimized for this type of adsorption studies, whereas the allatom model is a universal force field aimed at a myriad of different applications. To obtain the same degree of accuracy for the all-atom model as for the united atom model would require a dedicated optimization of the all-atom model parameters. Such an optimization will be cumbersome, for it is our impression that the physical information required for such an optimization is not experimentally available in sufficient detail. Thus, it is not straightforward to obtain a physically realistic value for, e.g., the ratio of the size parameters for $\mathrm{C}$ and $\mathrm{H}$ atoms. Optimization of the all-atom model will be more difficult commensurate with its higher level of detail and sophistication, even if the pertinent information were available. It requires finetuning a larger number of parameters and, accordingly, a significantly larger experimental data set than is needed for the united atom model. In our opinion, the currently available experimental data suffice to optimize the united atom model but not the all-atom model.

The force field proposed here is primarily designed to reproduce thermodynamic properties of guest molecules in a host system at minimal computational cost. The internal structure of the guests and the guest-guest interactions are of less importance because the properties are dominated by the strong interaction with the force field exerted by the host. Adsorption in charge neutral structures takes place at sites with little or no electric field. For these reasons, the united atom model ${ }^{10}$ seems the most straightforward choice. We consider the $\mathrm{CH}_{x}$ groups as single, chargeless interaction centers with their own effective potentials. The beads in the chain are connected by harmonic
TABLE 1: Unit Cells Used in the Simulation ${ }^{a}$

\begin{tabular}{ccccccccc}
\hline & & \multicolumn{3}{c}{ cells } & & \multicolumn{3}{c}{ unit cell size $[\AA]$} \\
\cline { 3 - 4 } \cline { 7 - 9 } framework & density $\left[\mathrm{kg} / \mathrm{m}^{3}\right]$ & $x$ & $y$ & $z$ & & $x$ & $y$ & $z$ \\
\hline MFI & 1796.358 & 2 & 2 & 4 & & 40.044 & 39.798 & 53.532 \\
TON & 1968.733 & 3 & 3 & 7 & 41.577 & 52.260 & 35.266 \\
AFI & 1729.848 & 2 & 3 & 5 & & 47.548 & 41.178 & 42.420 \\
DDR & 1759.963 & 2 & 3 & 1 & & 48.012 & 41.580 & 40.892 \\
MWW & 1673.460 & 1 & 2 & 1 & & 24.447 & 28.228 & 24.882
\end{tabular}

${ }^{a}$ For convenience the crystallographic cells are converted to orthorhombic cells.

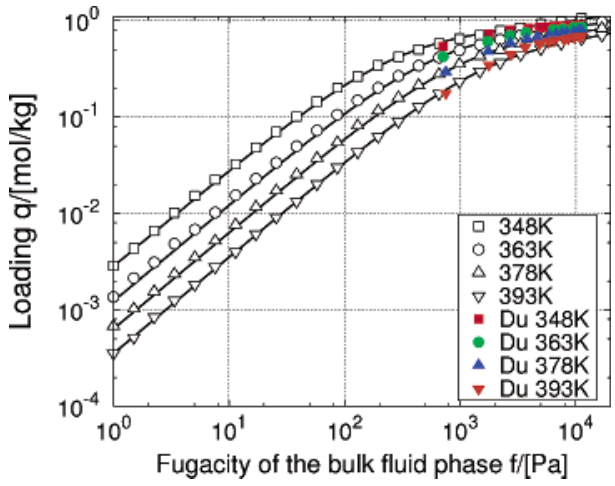

Figure 1. Isotherm of $n$-hexane in MWW at various temperatures. The experimental data are taken from Du et al. ${ }^{49}$

bonding potentials. A harmonic cosine bending potential models the bond bending between three neighboring beads, and a Ryckaert-Bellemans potential controls the torsional angle. The beads in a chain separated by more than three bonds interact with each other through a Lennard-Jones potential. The LennardJones potentials are shifted and cut at $12 \AA$. Analytical tailcorrections do not apply in zeolites. ${ }^{5}$ A truncated and shifted potential is equally suitable to Monte Carlo and molecular dynamics. Flexibility of the framework is not an issue for adsorption of linear and branched alkanes. ${ }^{11}$ The interactions between the rigid framework and the guest molecules are assumed to be dominated by the oxygen atoms. ${ }^{12}$ We have used the crystallographic structures of van Koningsveld et al., ${ }^{13}$ Marler, ${ }^{14}$ Qiu et al., ${ }^{15}$ Gies, ${ }^{16}$ and Camblor et al. ${ }^{17}$ The used unit cells and their sizes are listed in Table 1.

The conventional simulation techniques to compute adsorption isotherms are prohibitively expensive for long alkanes. The configurational bias Monte Carlo (CBMC) technique simulates the adsorption isotherms at affordable cost. ${ }^{18}$ In a CBMC simulation, chains are grown bead by bead biasing the growth process toward energetically favorable configurations, and avoiding overlap with the zeolite. During the growth, the Rosenbluth factor is calculated. The average Rosenbluth factor is directly related to the excess chemical potential, the free energy, and the Henry coefficient $K_{\mathrm{H}}{ }^{19,20}$ The CBMC algorithm greatly improves the conformational sampling of molecules and increases the efficiency of chain insertions by many orders of magnitude. More details on the simulations can be found in refs 7, 19, and 20 and in the Appendix.

B. Selection of Experimental Datasets. The parameters in current force fields for adsorption in porous media are usually tuned to reproduce heats of adsorption and Henry coefficients. However, it is difficult to identify unambiguously correct physical values for these parameters. Figure 1 illustrates the problem. It shows the experimentally determined $n$-hexane adsorption by a MWW-type zeolite along with our prediction from simulation. The loading is directly proportional to the pressure only at the extremely low pressures in the Henry 


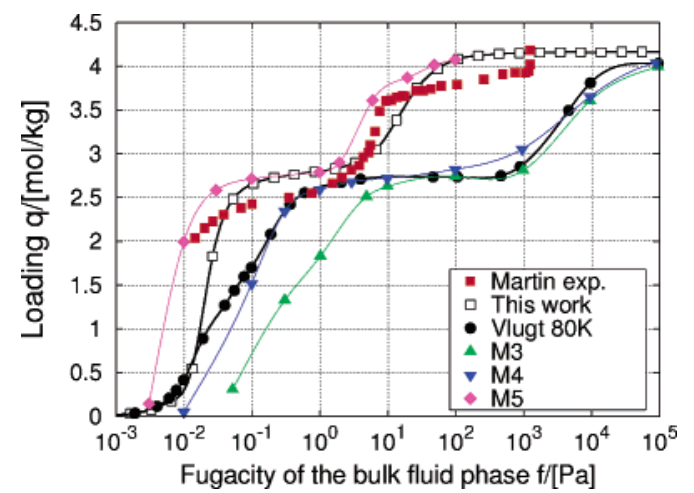

Figure 2. Isotherm of methane at $77 \mathrm{~K}$ in an aluminophosphate $\mathrm{AlPO}_{4}-5$ (AFI-topology). The experimental data are taken from Martin et al., ${ }^{21}$ the M3, M4, and M5 simulation data are from from ref 8 , and the simulation data of Vlugt are from ref 65.

regime. When plotted on a log-log scale, it becomes apparent that most available experimental isotherms are not inside but outside the Henry regime. Experimentally, it is quite difficult to obtain reliable measurements at very low pressures. Usual experimental procedures to obtain Henry coefficients involve fitting the measured data with an equation for an isotherm, followed by extrapolation to zero pressure and loading. In the absence of actual low pressure data, this introduces significant errors. The margin for error increases further, when the heats of adsorption are determined from the temperature dependence of the Henry coefficients. Our results strongly indicate that in many instances extrapolation to zero loading was not justified, because of a lack of low-pressure data, because of a lack of high-pressure data, or because there were altogether too few experimental data points.

A better approach would be to fit on entire isotherms. However, several problems arise. At very high pressures (to determine the saturation loading), a commonly occurring experimental difficulty is that adsorption is not restricted to the pores defined by the framework topology under investigation but also occurs at the exterior crystal surface. Since the texture of the crystals and crystal agglomerates varies widely, the maximum loading reported in the literature tends to show a wide scatter. An example is methane in tubular AFI-like structures. Figure 2 shows the isotherm of methane in an AFI-type aluminophosphate at $77 \mathrm{~K}$. AFI-type structures consist of straight, nonintersecting channels that are $0.73 \mathrm{~nm} \times 0.73 \mathrm{~nm}$ in diameter. The experimental results of Martin et al. ${ }^{21}$ illustrate a problem frequently encountered when trying to link experiments on the AFI-type pores to simulation. Simulation uses perfect crystals, whereas the pores in the actual samples used by Martin are (partially) blocked. Due to the one-dimensional character, a very small structural imperfection can block off a large part of the zeolite. In fact, Martin et al. studied several samples of different origin and found significantly different adsorption capacities. The authors estimate the ideal sorption capacity at 6 molecules per unit cell $(4.16 \mathrm{~mol} / \mathrm{kg})$, which matches our maximum loading from simulation. At $1000 \mathrm{~Pa}$, condensation on the external surface intrudes the experimental measurements, whereas the simulation uses fugacity and is not hampered by this transition from gas to liquid-phase adsorption.

C. Parameter Optimization Strategy. Instead of calibrating a force field with extrapolated experimental data, we propose to calibrate it by explicitly fitting the entire isotherm over a wide range of pressures and temperatures. If this procedure were followed for individual molecules, it would not necessarily yield a consistent force field, for many different sets of model
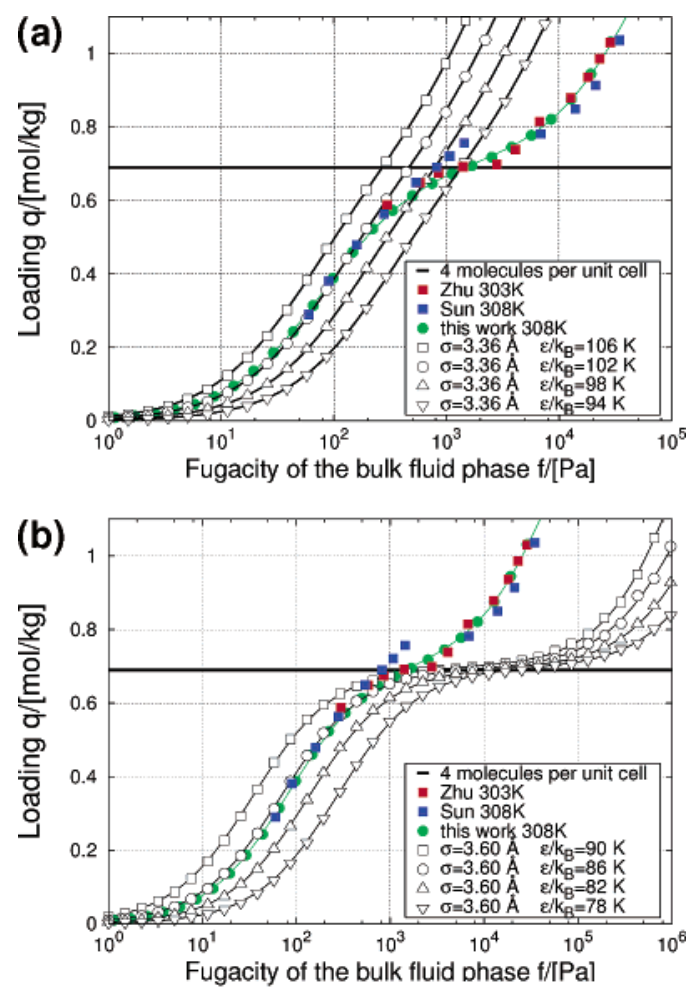

Figure 3. Isotherms of 2-methylpropane at $308 \mathrm{~K}$ in MFI. The $\mathrm{O}-\mathrm{CH}$ parameters remain fixed at $\sigma=3.92 \AA$ and $\epsilon / k_{\mathrm{B}}=40 \mathrm{~K}$, whereas $\epsilon_{\mathrm{O}-\mathrm{CH}_{3}}$ is examined over a range of reasonable values for two fixed values of $\sigma_{\mathrm{O}-\mathrm{CH}_{3}}(\mathrm{a})$ a rather too small of $\sigma_{\mathrm{O}-\mathrm{CH}_{3}}=3.36 \AA$ and (b) a too high value of $\sigma_{\mathrm{O}-\mathrm{CH}_{3}}=3.60 \AA$. Only a single parameter pair, $\epsilon_{\mathrm{O}-\mathrm{CH}_{3}} / k_{\mathrm{B}}=$ 93 and $\sigma_{\mathrm{O}-\mathrm{CH}_{3}}=3.48$ combined with the $\mathrm{CH}$ parameters (Table 2 ), is able to describe the experimental data of Sun et al. ${ }^{36}$ and Zhu et al. ${ }^{33}$

parameters are able to properly reproduce one and the same isotherm. A necessary and sufficient procedure is to utilize isotherms that exhibit inflection points and use these inflection points as calibration points for the parameter optimization.

It is instructive to discuss the role of the size parameter $\sigma_{\mathrm{O}-\mathrm{CH}_{x}}$. In Figure 3, we show the influence of the $\sigma$ parameters on the inflection of 2-methylpropane in MFI. The $\mathrm{O}-\mathrm{CH}$ parameters remain fixed at $\sigma=3.92 \AA$ and $\epsilon / k_{\mathrm{B}}=40 \mathrm{~K}$, whereas $\epsilon_{\mathrm{O}-\mathrm{CH}_{3}}$ is examined over a range of reasonable values for two values of $\sigma_{\mathrm{O}-\mathrm{CH}_{3}}$ : one significantly too small and one significantly too large. A crucial observation is that only a single strength/size parameter pair is able to describe the inflection and the entire isotherm properly. This is in contrast with the common belief that for each value of $\sigma$ there is a corresponding $\epsilon$ that can decribe the isotherm correctly. ${ }^{22}$ The shape of the isotherm and the inflection points are the most sensitive to the size parameter of the interactions, whereas the loading at a given pressure is most sensitive to the strength parameter of the interaction. A higher strength parameter $\epsilon$ induces an increased loading, and a lower strength parameter results in a decrease in loading (for a fixed pressure). The amount of inflection is controlled by the size parameter $\sigma$. These properties can be exploited to obtain unique parameters.

In practice, we proceed as follows. A reasonable starting size parameter is chosen. For this parameter, we iteratively search for the corresponding strength parameter that matches the experimental data at a pressure significantly below the inflection. The entire isotherm is then followed for increasing pressure until a deviation from the experimental data is observed. The "updated" size parameter is then found by choosing a higher value for a deviation to the left of the experimental data and by choosing a lower value for the size parameter for a deviation 


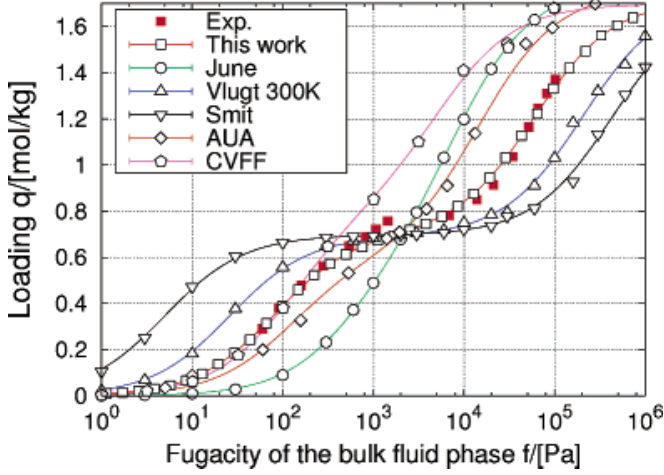

Figure 4. Isotherms of 2-methylpropane at $308 \mathrm{~K}$ in MFI compared to various computational models. The experimental data are taken from ref 36, the simulation data from June et al., ${ }^{23}$ Vlugt et al., ${ }^{7}$ Smit et al., ${ }^{25}$ AUA from Pascual et al., ${ }^{24}$ and CVFF from Macedonia et al. ${ }^{5}$

TABLE 2: Adsorbent-Adsorbate Interaction Size Parameters $\sigma$ and Strength Parameters $\epsilon$ Used in Various United Atom Models

\begin{tabular}{|c|c|c|c|c|c|c|}
\hline \multirow[b]{2}{*}{ model } & \multicolumn{2}{|c|}{$\mathrm{O}-\mathrm{CH}_{3}$} & \multicolumn{2}{|c|}{$\mathrm{O}-\mathrm{CH}_{2}$} & \multicolumn{2}{|c|}{$\mathrm{O}-\mathrm{CH}$} \\
\hline & $\overline{\sigma[\AA]}$ & $\epsilon / k_{\mathrm{B}}[\mathrm{K}]$ & $\overline{\sigma[\AA]}$ & $\overline{\epsilon / k_{\mathrm{B}}[\mathrm{K}]}$ & $\overline{\sigma[\AA]}$ & $\overline{\epsilon / k_{\mathrm{B}}[\mathrm{K}]}$ \\
\hline AUA & 3.30 & 106 & 3.23 & 89.84 & 3.18 & 69.05 \\
\hline June et al. & 3.364 & 83.8 & 3.364 & 83.8 & & \\
\hline this work & 3.48 & 93 & 3.58 & 60.5 & 3.92 & 40 \\
\hline Vlugt et al. & 3.60 & 80 & 3.60 & 58 & 3.60 & 58 \\
\hline Smit et al. & 3.64 & 87.5 & 3.64 & 54.4 & 3.64 & 51.3 \\
\hline
\end{tabular}

to the right of the experimental data. This scheme proceeds iteratively until the entire experimental isotherm is accounted for.

In Figure 4, we show the influence of the $\sigma$ parameter on the inflection of 2-methylpropane in MFI. Although the size parameters listed in Table 2 differ by less than $10 \%$, the shape of the isotherms is dramatically different. The model of June et al. $^{23}$ uses a small value of $\sigma=3.364 \AA$, and the AUA-model ${ }^{24}$ uses $\sigma_{\mathrm{O}-\mathrm{CH}_{3}}=3.30 \AA, \sigma_{\mathrm{O}-\mathrm{CH}_{2}}=3.23 \AA$, and $\sigma_{\mathrm{O}-\mathrm{CH}}=3.18 \AA$. The models of Vlugt et al. ${ }^{7}$ and Smit et al. ${ }^{25}$ use a fixed $\sigma$; $\sigma_{\mathrm{O}-\mathrm{CH}_{3}}=\sigma_{\mathrm{O}-\mathrm{CH}_{2}}=\sigma_{\mathrm{O}-\mathrm{CH}}=3.60 \AA$ for the Vlugt model and $\sigma_{\mathrm{O}-\mathrm{CH}_{3}}=\sigma_{\mathrm{O}-\mathrm{CH}_{2}}=\sigma_{\mathrm{O}-\mathrm{CH}}=3.64 \AA$ for the Smit model. The model proposed in this work uses $\sigma_{\mathrm{O}-\mathrm{CH}_{3}}=3.48 \AA$, $\sigma_{\mathrm{O}-\mathrm{CH}_{2}}=$ $3.58 \AA$, and $\sigma_{\mathrm{O}-\mathrm{CH}}=3.92 \AA$. It yields exact overlap with experimental data and the inflection is reproduced faithfully. In the remainder of this paper we will demonstrate their accuracy.

The fitting to well-established inflection points in the isotherms has many advantages and overcomes problems that have so far impeded the development of more accurate force fields.

(1) We obtain a unique set of parameters that all relate directly to a well-defined physical property. We therefore expect these parameters to be much more transferable to other systems than previous attempts.

(2) The parameters are determined accurately. The inflection in an isotherm is extremely sensitive to the size parameter $\sigma_{\mathrm{O}-\mathrm{CH}_{x}}$

(3) By explicitly fitting to entire adsorption isotherms we guarantee the proper reproduction of properties such as Henry coefficients, heats of adsorption, adsorption entropies, and maximum loadings.

(4) Inflections are found at moderate pressures and here the experimental data are most reliable. Experimentally there is minimal intrusion from adsorption at the exterior surface.
(5) The inflection is directly related to the structure e. g. for $n$-heptane and 2-methylpropane in MFI the inflection occurs exactly at 4 molecules per unit cell.

D. Parameters from MFI/AFI Inflections. The isotherms measured on MFI are optimally suited for calibration of a force field, because they have been reported by many different experimental research groups, and the fundamental reason for their shapes is very well established. The MFI-type structure consist of a three-dimensional pore system with straight, parallel channels intersected by zigzag channels. The linear channels intersect with the zigzag channels four times per unit cell. Interestingly, for $n$-hexane, $n$-heptane, and the branched alkanes in MFI, a kink in the isotherm is observed. ${ }^{7}$ This inflection is directly related to the number of intersections in the structure and occurs at exactly four molecules per unit cell. The fundamental understanding of the inflection points affords an independent check on the consistency of experimental data. If isotherms do not show an inflection point at the correct loading they can be summarily excluded.

Ethane, $n$-heptane, and 2-methylpropane exhibit isotherms of the Brunauer type-VI in MFI. Ethane shows a small inflection point in the adsorption isotherm at high loading. ${ }^{26}$ The $\epsilon_{\mathrm{O}-\mathrm{CH}_{3}}$ and $\sigma_{\mathrm{O}-\mathrm{CH}_{3}}$ are uniquely obtainable from the ethane isotherm. When the channel interiors are occupied, the probability distribution shows a remarkable order: a repeating pattern of ethane molecules "locked" in the zigzag channels between two intersections. The $\epsilon_{\mathrm{O}-\mathrm{CH}_{2}}$ and $\sigma_{\mathrm{O}-\mathrm{CH}_{2}}$ are obtained from $n$ heptane. The inflection behavior of $n$-heptane is well established. ${ }^{18,27}$ Smit and Maesen explained this effect in terms of commensurate freezing: $n$-heptane has a size commensurate with the size of the zigzag channel. At high pressures, the molecules shift from a random distribution to a distribution where the molecules are localized exclusively in the channels and not at the intersections. Various branched molecules show inflections for another reason. ${ }^{7}$ 2-Methylpropane preferentially adsorbs at the intersections. At a loading of four molecules per unit cell, the intersections are fully occupied, and additional molecules must be pushed into the channels requiring a significantly higher driving force. ${ }^{28}$ The $\epsilon_{\mathrm{O}-\mathrm{CH}}$ and $\sigma_{\mathrm{O}-\mathrm{CH}}$ are uniquely obtainable from the isotherm of 2-methylpropane. Detailed inspection of the experimental data showed that for ethane, 2-methylpropane, and $n$-heptane several independent groups provided consistent data, and we used these data as our primary set of experimental data. As basis for calibration, we utilized the experimental data from several different research groups of Cavalcante et al., ${ }^{29}$ Jolimaitre et al., ${ }^{30,31}$ Eder et al., ${ }^{32}$ Zhu et al., ${ }^{33-35}$ Sun et al., ${ }^{27,36}$ and Choudhary et al. ${ }^{37}$

Whereas inflection points in the isotherms of MFI-type zeolites can be used to calibrate most of the parameters, it does not afford calibration of the parameters for $\mathrm{CH}_{4}$. For this molecule, we resorted to AFI-type sieves. The isotherms for $\mathrm{CH}_{4}$ at $77 \mathrm{~K}$ have a clearly defined inflection point at 4 molecules per unit cell $(2.77 \mathrm{~mol} / \mathrm{kg})$ loading. Therefore $\epsilon_{\mathrm{O}-\mathrm{CH}_{4}}$ and $\sigma_{\mathrm{O}-\mathrm{CH}_{4}}$ are obtained from the isotherm of methane in AFI. There are no experimental isotherms of double branched alkanes with an inflection, so that the $\epsilon_{\mathrm{O}-\mathrm{C}}$ and $\sigma_{\mathrm{O}-\mathrm{C}}$ could not be uniquely and accurately determined. Their initial values had to be estimated from mixing rules. Calibration of these values utilizing an entire isotherm of 2,2-dimethylbutane in MFI indicated that the initial estimates were essentially correct. The resulting force field is described by the parameters listed in Table 3.

E. Comparing This Work and Calibration Data. The inflection of methane in AFI at $77 \mathrm{~K}$ is found at the experimental 
TABLE 3: Force Field Guest-Host and Guest-Guest Interactions of Hydrocarbons in Charge Neutral Nanoporous Materials ${ }^{a}$

\begin{tabular}{|c|c|c|c|c|c|c|}
\hline & $\mathrm{O}$ & $\mathrm{CH}_{4}$ & $\mathrm{CH}_{3}$ & $\mathrm{CH}_{2}$ & $\mathrm{CH}$ & $\mathrm{C}$ \\
\hline \multirow[t]{2}{*}{$\mathrm{CH}_{4}$} & 115.00 & 158.50 & 130.84 & 94.21 & 51.91 & 11.26 \\
\hline & 3.47 & 3.72 & 3.74 & 3.84 & 4.17 & 4.87 \\
\hline \multirow[t]{2}{*}{$\mathrm{CH}_{3}$} & 93.00 & 130.84 & 108.00 & 77.77 & 42.85 & 9.30 \\
\hline & 3.48 & 3.74 & 3.76 & 3.86 & 4.19 & 4.90 \\
\hline \multirow[t]{2}{*}{$\mathrm{CH}_{2}$} & 60.50 & 94.21 & 77.77 & 56.00 & 30.85 & 6.69 \\
\hline & 3.58 & 3.84 & 3.86 & 3.96 & 4.30 & 5.03 \\
\hline \multirow[t]{2}{*}{$\mathrm{CH}$} & 40.00 & 51.91 & 42.85 & 30.85 & 17.00 & 3.69 \\
\hline & 3.92 & 4.17 & 4.19 & 4.30 & 4.67 & 5.46 \\
\hline \multirow[t]{2}{*}{$\mathrm{C}$} & 10.00 & 11.26 & 9.30 & 6.69 & 3.69 & 0.80 \\
\hline & 4.56 & 4.87 & 4.90 & 5.03 & 5.46 & 6.38 \\
\hline
\end{tabular}

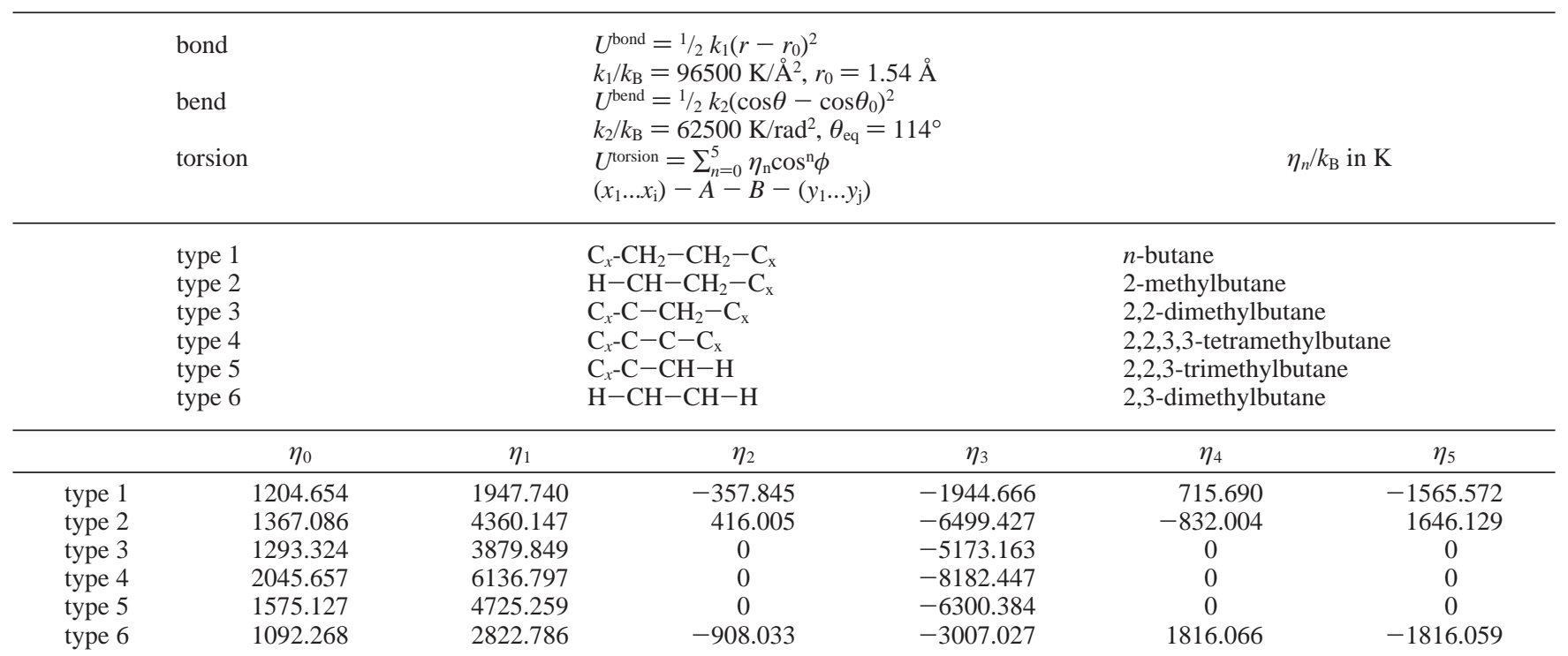

${ }^{a}$ Lennard-Jones parameters, $\epsilon / k_{\mathrm{B}}[\mathrm{K}]$ in top, $\sigma[\AA]$ in bottom of each field, bond and bend parameters, and the torsion potential: the torsion type on the left, on the right an example of a molecule with this type of torsion potential, and on the bottom the parameters. Some of the alkane-alkane interactions are taken from ref 66 and optimized to reproduce vapor-liquid coexistence curves of the phase diagrams, the internal bond from ref 67 , the internal bend from ref 41 , and the torsion from T. J. H. Vlugt and M. Frash. ${ }^{68}$

pressure, and the isotherm shape is satisfactorily reproduced (Figure 2). The $\epsilon_{\mathrm{O}-\mathrm{CH}_{4}}$ and $\sigma_{\mathrm{O}-\mathrm{CH}_{4}}$ could be uniquely determined, with an accuracy better than $0.02 \AA$ for $\sigma$ and better than $5 \mathrm{~K}$ for $\epsilon / k_{\mathrm{B}}$. Figure 5 shows the results of the fitting procedure of ethane and $n$-heptane in MFI along with the experimental basis set. The $\epsilon_{\mathrm{O}-\mathrm{CH}_{3}}$ and $\sigma_{\mathrm{O}-\mathrm{CH}_{3}}$ parameters are uniquely fixed with a precision better than $0.01 \AA$ for $\sigma$ and better than $1 \mathrm{~K}$ for $\epsilon / k_{\mathrm{B}}$. The simulation results for ethane are in excellent agreement with the experimental data from Choudhary et al. (Figure 5a). The agreement with the data from Zhu et al. and Sun et al. is fair, for the former deviate at low pressures and the latter at high pressures. Considering the good agreement between the simulations and experiments, the results may be interpreted as indirect evidence for the ethane inflection, even though the experimental high pressure confirmation is missing. Normal heptane has a much more pronounced inflection behavior (Figure 5b). The $\epsilon_{\mathrm{O}-\mathrm{CH}_{2}}$ and $\sigma_{\mathrm{O}-\mathrm{CH}_{2}}$ are uniquely fixed with a precision better than $0.02 \AA$ for $\sigma$ and better than $5 \mathrm{~K}$ for $\epsilon / k_{\mathrm{B}}$. The simulated isotherms overlap perfectly with data of Eder et al. and well with the data of Sun et al. The few high pressure points of Sun et al. at $303 \mathrm{~K}$ are in disagreement with the simulations and with most experimental data on maximum loadings (1.25 mol/kg Yang and Rees ${ }^{38}$ and $1.265 \mathrm{~mol} / \mathrm{kg}$ van Well et al. ${ }^{39}$ )

The 2-methylpropane isotherms are compared in Figure 6a to the data of Sun et al. and Zhu et al. The agreement is again excellent, except for the low pressure part of the Sun data for $277 \mathrm{~K}$. The experimental loadings are probably too high because the inflection is expected at 4 molecules per unit cell $(0.6935$ $\mathrm{mol} / \mathrm{kg})$. The $\epsilon_{\mathrm{O}-\mathrm{CH}}$ and $\sigma_{\mathrm{O}-\mathrm{CH}}$ are uniquely fixed with a precision better than $0.01 \AA$ for $\sigma$ and better than $1 \mathrm{~K}$ for $\epsilon / k_{\mathrm{B}}$. Figure $6 \mathrm{~b}$ shows the double branched 2,2-dimethylbutane isotherm. The simulation data overlaps with Jolimaitre et al., and Cavalcante and Ruthven.

F. Comparing This Work and Preceding Models. To show the improvement of this work compared to previous approaches, we refer again to Figure 2. The figure shows another important point. Our approach clearly outperforms complex all-atom models containing two-and three-body dispersion interactions between guest and framework atoms (up to quadrupole terms), induced interactions (polarization), and repulsive terms. As an example, the M3, M4, and M5 models are taken from ref 8. These three models differ only by a slight change in repulsive interaction. The M5 model is the best of the three but not better than our significantly less complex united atom approach. The success of the united atom model supports the notion that adsorption properties are dominated by dispersive forces and that a united atom model captures these satisfactorily.

We also refer again to Figure 4 to discuss the comparison with various united atom approaches previously proposed in the literature. The figure showed the inflection in the isotherm of 2-methylpropane at $308 \mathrm{~K}$ in MFI. The models of Smit et al. and Vlugt et al. exaggerated the inflections because their size parameters were too large. The models of Pascual et al. and June et al. and the all-atom CVFF force field did not show a clear inflection at all because their size parameters were too small.

The value of $\sigma_{\mathrm{O}-\mathrm{CH}_{x}}$ also has an effect on the maximum loading and packing efficiency. De Meyer et al. ${ }^{40}$ performed both experiments and simulations of long chain $n$-alkanes in 

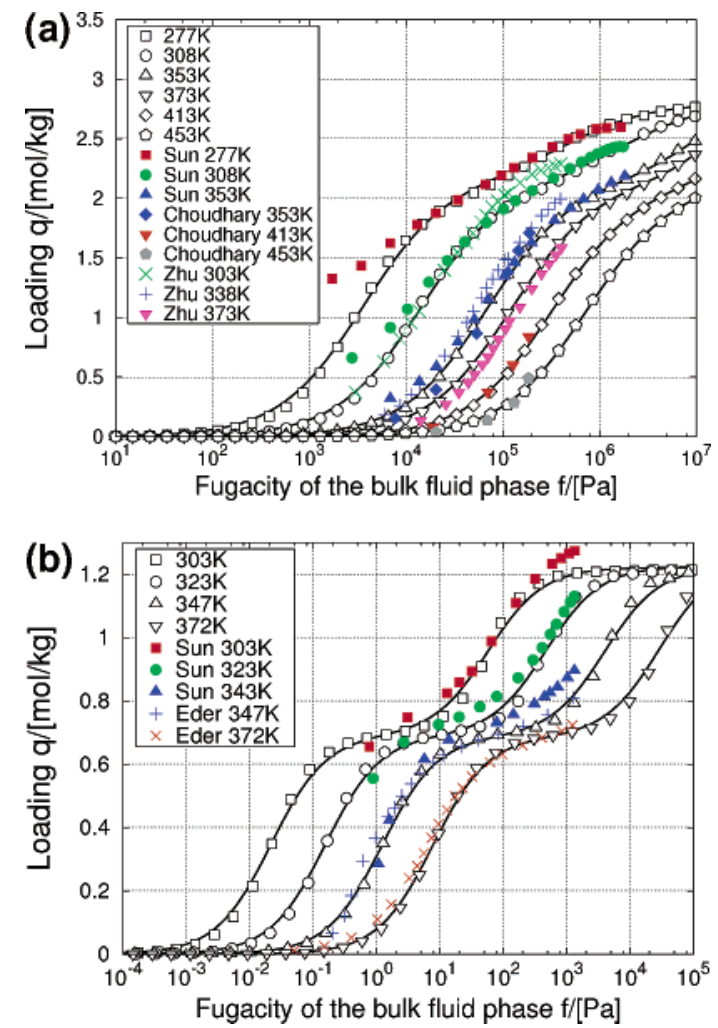

Figure 5. Isotherms of linear alkanes (a) ethane, and (b) $n$-heptane in MFI at various temperatures. Experimental data are taken from Sun et al., ${ }^{27,36}$ Choudhary et al., ${ }^{37}$ Eder et al., ${ }^{32}$ and Zhu et al. ${ }^{33,35}$
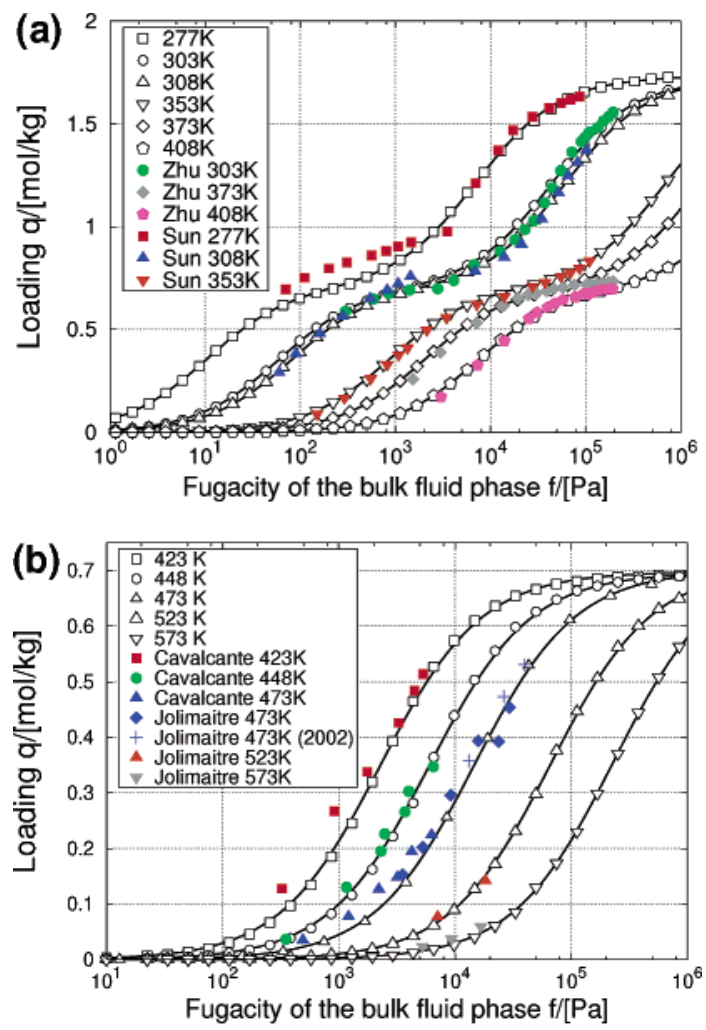

Figure 6. Isotherms of branched alkanes used in the calibration set of the force field (a) 2-methylpropane and (b) 2,2-dimethylbutane in MFI at various temperatures. Experimental data are taken from Sun et al., ${ }^{27,36}$ Zhu et al., ${ }^{33,35}$ Cavalcante et al., ${ }^{29}$ and Jolimaitre et al. ${ }^{30,31}$

MFI. Experiments show that the maximum packing is approximately 53.2 carbon atoms per unit cell for $n-\mathrm{C}_{14}$ and longer $n$-alkanes, whereas simulations using the model of Vlugt et al.

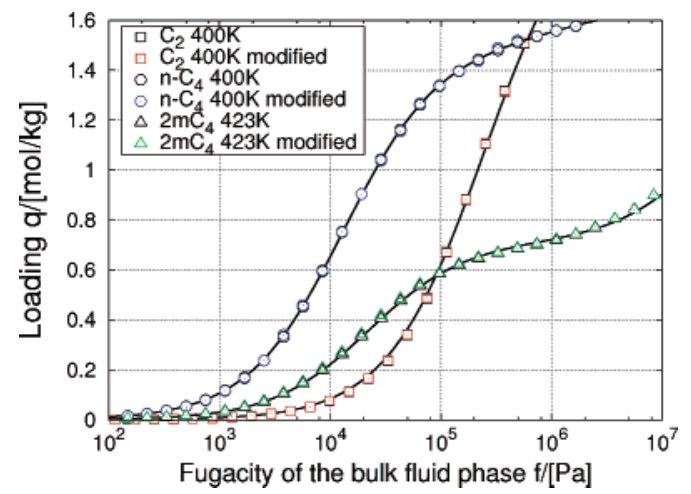

Figure 7. Isotherms of ethane, butane, and 2-methylbutane compared to isotherms obtained using different internal interaction potentials and parameters as described in the text.

find a value of 49.0 carbon atoms per unit cell. The current model yields 52.5 carbon atoms per unit cell in excellent agreement with experiment, but not with the model of Vlugt et al. This is another indication that the value for $\sigma_{\mathrm{O}-\mathrm{CH}_{x}}$ in the Vlugt model is too high.

G. Internal Interaction Parameters. To test the dependence of the adsorbent-adsorbate interaction parameters derived in this work on the internal interaction parameters, we apply different sets to ethane, butane, and 2-methylbutane and compare the isotherms with the loadings obtained using the internal interactions of this work (Table 3 ). We modified the harmonic bond potential to a fixed distance of $1.54 \AA$ and recomputed the isotherm of ethane. The butane model was modified to the TraPPE-UA model, ${ }^{41}$ which uses a fixed bond distance of 1.54 $\AA$, and a bending and torsion potential of the form

$$
U^{\text {bend }}=\sum_{\text {bends }} \frac{1}{2} k_{\theta}\left(\theta-\theta_{0}\right)^{2}
$$

with $k_{\theta} / k_{\mathrm{B}}=62500 \mathrm{~K} / \mathrm{rad}^{2}, \theta_{0}=114^{\circ}$, and

$$
\begin{array}{r}
U^{\text {torsion }}=\sum_{\text {torsions }} \eta_{0}+\eta_{1}[1+\cos (\phi)]+\eta_{2}[1-\cos (2 \phi)]+ \\
\eta_{3}[1+\cos (3 \phi)]
\end{array}
$$

with $\eta_{n} / k_{\mathrm{b}}=\{0,335.03,-68.19,791.32\}$. Another possible combination of parameters applied to 2-methylbutane is a fixed bond length of $1.53 \AA$, a bending potential of the form eq 1 with $k_{\theta} / k_{\mathrm{B}}=85000 \mathrm{~K} / \mathrm{rad}^{2}, \theta_{0}=113^{\circ}$, and the torsion potential of this work. The results for the three test cases shown in Figure 7 suggest a minimal dependency of the adsorption results on the internal interaction parameters and that the adsorbentadsorbate interaction parameters may be combined with any other physically reasonable internal interaction model. The results are also largely independent of the intermolecular potentials, because these too are dominated by the adsorbentadsorbate interactions.

\section{Validation and Applications}

A. Extension To Other Sorbates in MFI. To demonstrate that our parameters are transferable to other molecules in MFI, we have selected methane, 2-methylbutane, 2-methylpentane, and 3-methylpentane. The simulated and experimental isotherms for methane on MFI are shown in Figure 8a. The agreement of the simulations and experiments is satisfactory considering the scatter in the experimental data sets. The temperature dependence, the amount adsorbed, and the shape of the isotherms are well reproduced. For 2-methylbutane (Figure 8b), we find 

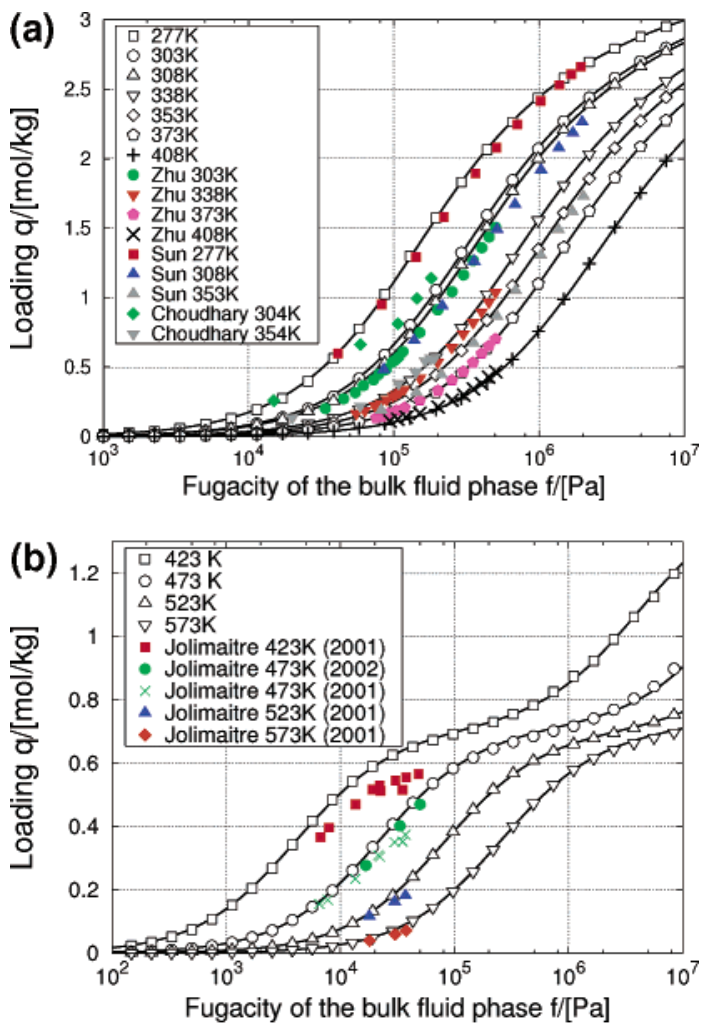

Figure 8. Isotherms of (a) methane and (b) 2-methylbutane in MFI at various temperatures. Experimental data are taken from Sun et al., ${ }^{36}$ Choudhary et al., ${ }^{37}$ and Jolimaitre et al..$^{30,31}$

excellent agreement with Jolimaitre et al. The data are obtained using pulse chromatography and uptake measurements and are in good agreement with each other. Once again we find a deviation at the lowest temperature. Reasons for deviations include adsorption in meso-pores and on the external surface, and at low temperatures, the sorption equilibration of particularly branched molecules materializes extremely slowly.

Figure 9a shows the computed isotherms for 2-methylpentane compared to Jolimaitre et al., Zhu et al., and Cavalcante et al. The discrepancy between the experimental sets is clearly visible. A likely cause for the difficulty in obtaining reliable data on 2-methylpentane is that the molecule is asymmetric and too long to easily change orientation at the intersections. For the more symmetric and smaller 2-methylbutane molecule this is less of a problem. The optimal packing at a certain pressure is hard to attain, in both experiment and simulation. The Cavalcante loading is too high in comparison with ours. The agreement with Jolimaitre is reasonable, although only one temperature is available. The data of Zhu et al. deviates at higher temperatures. For 3-methylpentane (Figure 9b), we find excellent agreement with Zhu et al. and Jolimaitre et al. Thus, the agreement between simulated and experimental data on the adsorption of molecules not part of the calibration set is remarkably good, especially when the disagreement between the experimental data from various sources is taken into consideration.

B. Extension To Mixtures in MFI. Binary mixtures represent a critical test for our force field. Figure 10 compares the loading of the individual components of a mixture of $n$-hexane and 2-methylpentane as a function of 2-methylpentane in the gas phase at $433 \mathrm{~K}$ and $6.6 \mathrm{kPa}$ as obtained by simulation with those obtained through experiments. ${ }^{42}$ The loadings of the individual components at fractional compositions zero and one correspond to the pure component values and agree well with the simulation results. The simulation results show no clear
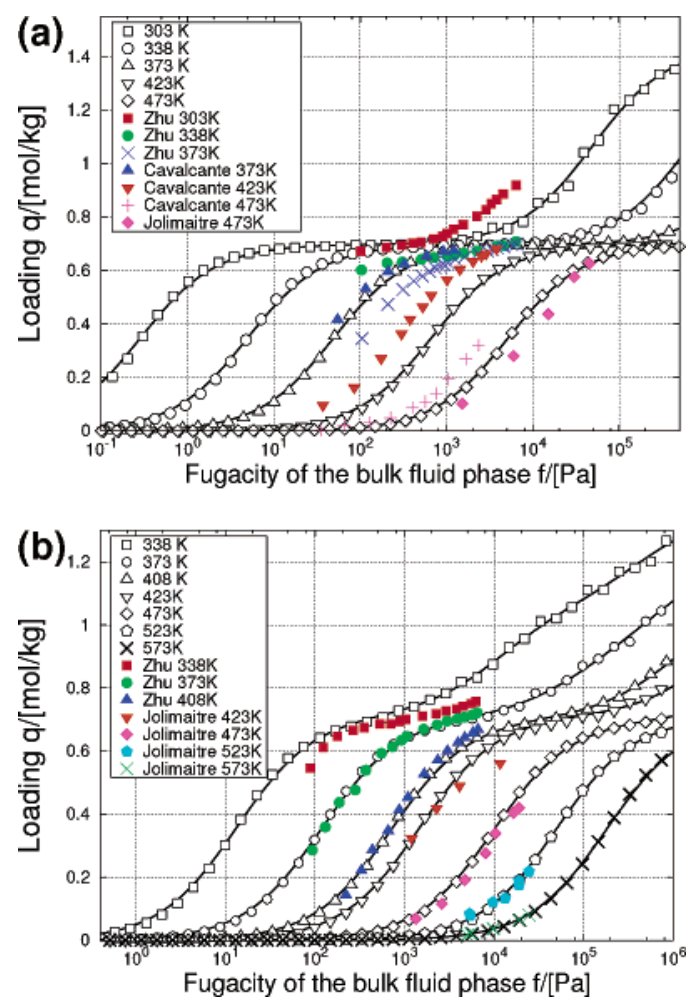

Figure 9. Isotherms of branched alkanes (a) 2-methylpentane and (b) 3-methylpentane in MFI at various temperatures. Experimental data are taken from Cavalcante et al., ${ }^{29}$ Jolimaitre et al., ${ }^{30}$ and Zhu et al. ${ }^{34}$

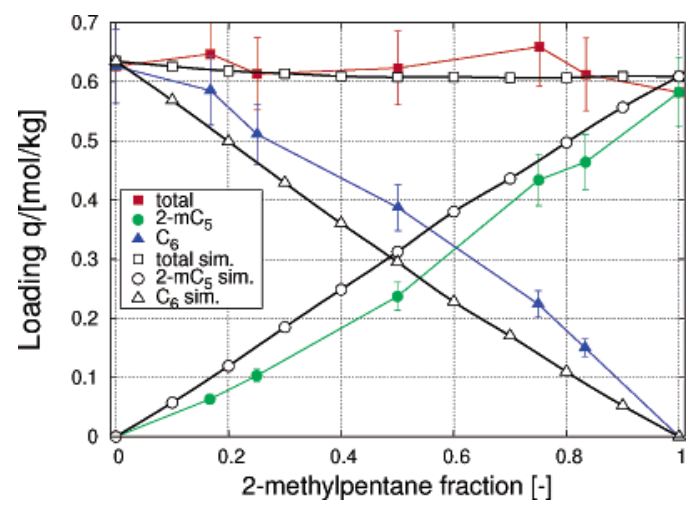

Figure 10. Hexane and 2-methylpentane loading in MFI as a function of 2-methylpentane fraction in the gas phase in a binary mixture at $433 \mathrm{~K}$ and $6.6 \mathrm{kPa}$. Experimental data are taken from Schuring et al. ${ }^{42}$

preference for either $n$-hexane or 2-methylpentane in this temperature and pressure region. The experimental results show a small preferential adsorption of $n$-hexane compared to 2-methylpentane. We note that the agreement with experiment is significantly improved compared to the model of Vlugt et al. Their model yielded a loading that is too high $(0.69 \mathrm{~mol} / \mathrm{kg}$ for $n$-hexane and $0.65 \mathrm{~mol} / \mathrm{kg}$ for 2-methylpentane) and a small preference for the branched instead of the experimentally preferred linear alkane. ${ }^{42}$ The pressure is too low to observe the exclusion effect of branched molecules compared to their linear isomers due to the configurational entropy effect. ${ }^{43}$

C. Extension To Low-Coverage in MFI. The force field developed thus far yields isotherm data that agree not only qualitatively, but also quantitatively with many experimental data sets, such as Sun et al., Jolimaitre et al., Choudhary et al., Zhu et al., and Eder et al. Surprisingly, the agreement between the experimental data and between simulated and experimental data breaks down at low coverage. This is especially striking because most of these data were obtained by extrapolating the 
TABLE 4: Comparison of Our Simulation Results of Low-Coverage Properties in MFI with the Experimental Results of Denayer et al. ${ }^{44} a$

\begin{tabular}{|c|c|c|c|c|c|c|}
\hline \multirow[b]{2}{*}{$\mathrm{CN}$} & \multicolumn{2}{|c|}{$K_{\mathrm{H}} 573 \mathrm{~K}[\mathrm{~mol} / \mathrm{kg} / \mathrm{Pa}]$} & \multicolumn{2}{|c|}{$K_{\infty}[\mathrm{mol} / \mathrm{kg} / \mathrm{Pa}]$} & \multicolumn{2}{|c|}{$-\Delta H[\mathrm{~kJ} / \mathrm{mol}]$} \\
\hline & sim. & exp. & sim. & exp. & sim. & exp. \\
\hline 5 & $3.04 \times 10^{-6}$ & $2.99 \times 10^{-6}$ & $2.33 \times 10^{-11}$ & $2.64 \times 10^{-11}$ & 56.13 & 55.7 \\
\hline 6 & $6.10 \times 10^{-6}$ & $5.93 \times 10^{-6}$ & $6.0 \times 10^{-11}$ & $6.07 \times 10^{-11}$ & 65.87 & 66.0 \\
\hline 7 & $1.23 \times 10^{-5}$ & $1.22 \times 10^{-5}$ & $1.53 \times 10^{-12}$ & $1.29 \times 10^{-12}$ & 75.77 & 76.7 \\
\hline 8 & $2.43 \times 10^{-5}$ & $2.49 \times 10^{-5}$ & $3.67 \times 10^{-13}$ & $3.25 \times 10^{-13}$ & 85.82 & 86.6 \\
\hline 9 & $4.61 \times 10^{-5}$ & $4.73 \times 10^{-5}$ & $8.59 \times 10^{-14}$ & $8.41 \times 10^{-14}$ & 95.81 & 96.1 \\
\hline \multicolumn{3}{|c|}{ relation } & \multicolumn{2}{|l|}{ sim. } & \multicolumn{2}{|c|}{ exp. } \\
\hline & $\begin{array}{l}=\alpha C N+\beta \\
=\gamma C N+\delta \\
(\infty)=-A \Delta H\end{array}$ & & $\begin{array}{l}=9.93 \\
=11.65 \\
=0.141, B=1\end{array}$ & & $\begin{array}{l}=10.1 \\
=11.99 \\
=0.143,\end{array}$ & \\
\hline
\end{tabular}

${ }^{a}$ Both the Denayer and the simulation Henry coefficients $K_{\mathrm{H}}$ of the linear alkanes have been fitted to $K_{\mathrm{H}}=K_{\infty} e^{-\Delta \mathrm{H} / \mathrm{RT}}$ in the temperature range $T=473-673 \mathrm{~K}$. Here, $K_{\infty}$ denotes the preexponential Henry coefficient, $\Delta H$ the enthalpy of adsorption, and $R=8.31451 \mathrm{~J} / \mathrm{mol} / \mathrm{K}$ the gas constant. The entropy $\Delta S$ per carbon number is related to the slope of $\ln \left(K_{\infty}\right)$ plotted as a function of Carbon Number $(\mathrm{CN}) .{ }^{44}$

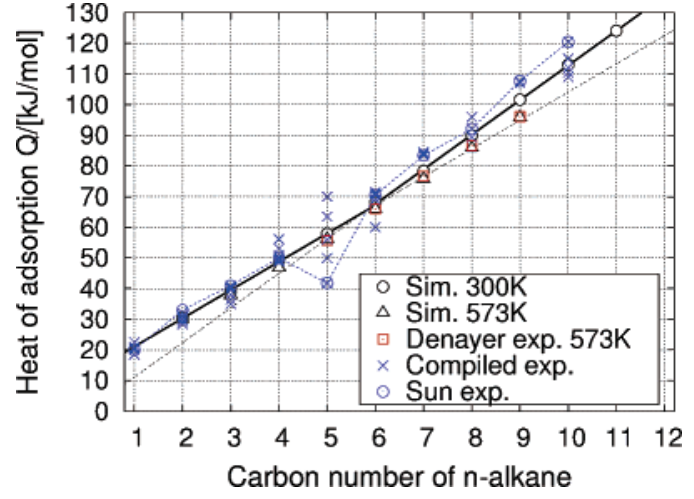

Figure 11. Comparison of computed heats of adsorption with experimental data for methane up to $n$-dodecane in MFI. Experimental data are taken from Sun et al..$^{27,36}$ and Denayer et al. ${ }^{44}$ and values found in the literature as compiled by refs 7 and 44 .

very same isotherms to low pressure and loading. An analysis of the experimental data reported by Denayer and co-workers ${ }^{44}$ sheds light on the likely reasons for these discrepancies. In marked contrast to other experimentalists, Denayer took special care to verify that the results were indeed obtained in the Henry regime.

In this section, we compare our simulation results with the experimental results from Denayer et al. on MFI. The results are summarized in Table 4. It is noteworthy that Denayer's data set was not part of the set used as a basis for our force field. The quantitative agreement and consistency on low-coverage properties of simulated and experimental data is therefore truly remarkable. We reproduce the chain length dependence of the enthalpy of adsorption and the entropy of adsorption, as well as the absolute values of Henry coefficients, preexponential factors, and enthalpies of adsorption.

A point of continued interest is the variation of the heat of adsorption with carbon number. Figure 11 shows this variation as obtained from simulation and from various experimental groups. Our results are consistent with Denayer et al. data at $573 \mathrm{~K}$ and also with other experimental data obtained around $300 \mathrm{~K}$. Sun et al. for $\mathrm{C}_{1}-\mathrm{C}_{12}$ derived his heats of adsorption from isotherms through extrapolation. These vary clearly in a more erratic fashion with carbon number than the data obtained through dedicated experiments at low pressure. A visual inspection of the simulated and most of the experimental data suggests that there are two linear correlations between the heat of adsorption and the Carbon Number $(\mathrm{CN})$, one for $\mathrm{C}_{1}-\mathrm{C}_{5}$ and another for $\mathrm{C}_{6}-\mathrm{C}_{12}$. Our simulation at $300 \mathrm{~K}$ indicates a slope of $9.22 \times \mathrm{CN}$ for $\mathrm{C}_{1}-\mathrm{C}_{5}$, and a slope of $11.3 \times \mathrm{CN}$ for
$\mathrm{C}_{6}-\mathrm{C}_{12}$. Various different values have been reported in the literature: $9.81,10.08,10.2,11.0,11.3$, and $12 \mathrm{~kJ} / \mathrm{mol}$ per carbon number. We note that simulation models of June et al. and Vlugt et al. do not resolve these two distinct regimes. The model of Smit et al. resolves two regimes with a crossover at $\mathrm{C}_{8}$ instead of $\mathrm{C}_{6}$.

Compared to linear alkanes, far fewer experimental data are available on the adsorption of branched alkanes in MFI. A detailed study of linear and branched alkanes in protonated MFI is available from Denayer et al. ${ }^{45}$ Despite the absence of protons in the simulated framework structure and the presence of protons in the experimental sample, the agreement between simulated and experimental Henry coefficients in Table 5 is fair. Both the simulations and the data of Denayer et al. agree on the ordering of the Henry coefficients for a set of isomers: linear $>$ 2-methyl > 3-methyl > dibranched. The same order applies to the heats of adsorption. Comparison between simulated and experimental heats of adsorption from sources other than Denayer and co-workers does not seem to be a meaningful endeavor, for the scatter in the experimentally data in Table 5 (compiled by refs 7 and 44) is huge.

The good match between simulated and a single set of experimental data outside our calibration set strongly suggests that the pulse chromatographic technique used by Denayer is uniquely suited to obtain reliable low coverage data and that extrapolation of isotherms from intermediate to low coverage tends to introduce major errors.

D. Extension To Different Topologies. Validation of our model for siliceous zeolites other than MFI relies on the relatively few data available for DDR, ${ }^{46,47} \mathrm{TON},{ }^{48}$ and MWW. ${ }^{49}$ The DDR topology consists of 19-hedron cavities connected through 8-ring windows of $0.35 \mathrm{~nm} \times 0.44 \mathrm{~nm}$ across into a hexagonally arranged two-dimensional cage/window-type systems. Figure 12 shows our simulation results for ethane compared with the experimental data of Zhu et al. for DDR. The agreement is excellent and we find overlap at all temperatures. The heat of adsorption computed at $300 \mathrm{~K}$ for ethane is $28.96 \mathrm{~kJ} / \mathrm{mol}$, whereas Zhu et al. found $24.74 \mathrm{~kJ} / \mathrm{mol}$ when he used the virial form of the thermodynamic equilibrium equation to extrapolate the data to low loading. The Henry coefficients obtained in this way are fitted to the van't Hoff equation to provide the heat of adsorption. However, a closer inspection of the data plotted at $\log -\log$ reveals that the data of Zhu et al. are too far outside the Henry regime to produce reliable results.

The TON topology consists of narrow, unidimensional 10ring channels with small apertures of $0.46 \mathrm{~nm} \times 0.57 \mathrm{~nm}$. Hampson and Rees measured adsorption data for ethane and 
TABLE 5: Comparison of Our Simulations Results of the Henry Coefficients $K_{\mathrm{H}}$ and Enthalpies of Adsorption $\Delta H$ of Linear and Branched Alkanes in MFI with the Experimental Results of Denayer and Co-workers on Protonated MFI ${ }^{45} a$

\begin{tabular}{cllcccc}
\hline $\mathrm{CN}$ & \multicolumn{1}{c}{ guest } & $\begin{array}{c}K_{\mathrm{H}} 573 \mathrm{~K} \mathrm{sim} . \\
{[\mathrm{mol} / \mathrm{kg} / \mathrm{Pa}]}\end{array}$ & $\begin{array}{c}K_{\mathrm{H}} 573 \mathrm{~K} \text { exp. } \\
{[\mathrm{mol} / \mathrm{kg} / \mathrm{Pa}]}\end{array}$ & $\begin{array}{c}-\Delta H 300 \mathrm{~K} \mathrm{sim} . \\
{[\mathrm{kJ} / \mathrm{mol}]}\end{array}$ & $\begin{array}{c}-\Delta H 573 \mathrm{~K} \text { exp. } \\
{[\mathrm{kJ} / \mathrm{mol}]}\end{array}$ & $\begin{array}{c}\text { values from literature } \\
{[\mathrm{kJ} / \mathrm{mol}]}\end{array}$ \\
\hline 5 & $n-\mathrm{C}_{5}$ & $3.04 \times 10^{-6}$ & $4.74 \times 10^{-6}$ & 57.93 & 57.7 & $60.0,64.5$ \\
& $2-\mathrm{mC}_{4}$ & $2.72 \times 10^{-6}$ & $3.34 \times 10^{-6}$ & 55.77 & 56.1 & $57.4,56.1,58.4$ \\
6 & $n-\mathrm{C}_{6}$ & $6.1 \times 10^{-6}$ & $9.73 \times 10^{-6}$ & 68.06 & 68.8 & $69.9,70.0,72.0,71.5$ \\
& $2-\mathrm{mC}_{5}$ & $5.98 \times 10^{-6}$ & $6.04 \times 10^{-6}$ & 67.88 & 66.8 & $67.8,58.5,64.0$ \\
& $3-\mathrm{mC}_{5}$ & $4.0 \times 10^{-6}$ & $5.23 \times 10^{-6}$ & 65.00 & 66.0 & $62.8,61.5,63.0,62.7,66.4,66.8,60.0$ \\
& $2,2-\mathrm{dmC}_{4}$ & $2.93 \times 10^{-6}$ & $3.33 \times 10^{-6}$ & 62.20 & 63.9 & $54.4,68.4,63.0,67.7,55.0,58.4,54.4$ \\
& $2,3-\mathrm{dmC}_{4}$ & $4.18 \times 10^{-6}$ & $3.12 \times 10^{-6}$ & 65.7 & 63.4 & 54.4 \\
7 & $n-\mathrm{C}_{7}$ & $1.23 \times 10^{-5}$ & $1.96 \times 10^{-5}$ & 78.32 & 79.6 & $82.6,84.0,84.5$ \\
& $2-\mathrm{mC}_{6}$ & $1.08 \times 10^{-5}$ & $1.10 \times 10^{-5}$ & 79.33 & 78.4 & \\
& $3-\mathrm{mC}_{6}$ & $9.46 \times 10^{-6}$ & $1.04 \times 10^{-5}$ & 77.00 & 78.0 & \\
& $2,3-\mathrm{dmC}_{5}$ & $5.5 \times 10^{-6}$ & $4.24 \times 10^{-6}$ & 76.18 & 74.1 & $96.0,100.7$ \\
& $n-\mathrm{C}_{8}$ & $2.43 \times 10^{-5}$ & $3.91 \times 10^{-5}$ & 89.95 & 90.7 & 89.0 \\
& $2-\mathrm{mC}_{7}$ & $2.10 \times 10^{-5}$ & $2.15 \times 10^{-5}$ & 88.75 & 88.6 & \\
& $3-\mathrm{mC}_{7}$ & $1.66 \times 10^{-5}$ & $1.8 \times 10^{-5}$ & 88.27 & 88.5 &
\end{tabular}

${ }^{a}$ The values for the heat of adsorption are taken from refs 7 and 44 .

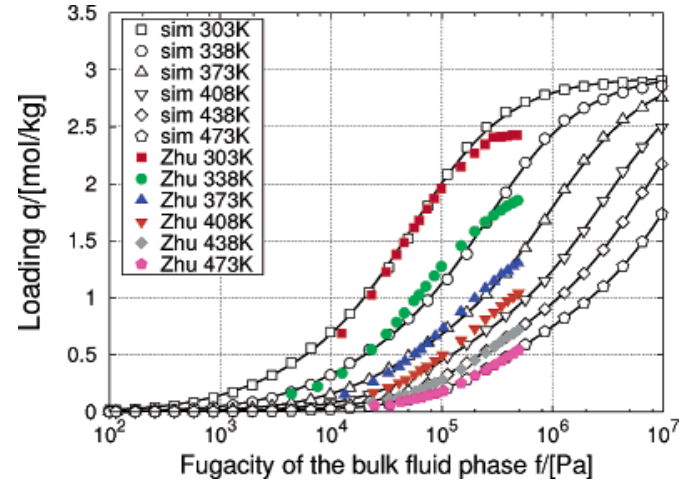

Figure 12. Isotherm of ethane in DDR at various temperatures. Experimental data are taken from $\mathrm{Zhu}$ et al. ${ }^{46}$

propane on TON. ${ }^{48}$ Our simulation data and the experimental data are in excellent agreement. For $\mathrm{C}_{2}$ and $\mathrm{C}_{3}$, the simulated (32.0 and $41.6 \mathrm{~kJ} / \mathrm{mol}$ ) and experimental heat of adsorption (31.9 and $42.0 \mathrm{~kJ} / \mathrm{mol}$ ) are virtually identical. If framework flexibility were to be important, it would be in this highly confined environment. The agreement of simulation utilizing the model with a completely fixed framework corroborates earlier suggestions that framework flexibility does not significantly influence the adsorption properties, even in tight confinements. For a comparison of the heat of adsorption obtained from simulations based on TON and those obtained from experiments on TON aluminosilicates, the differences are apparent, particularly for longer alkanes. These are probably caused by adsorption on the Brönsted acid sites. ${ }^{50}$ For TON zeolite with a Si/Al ratio of 30 , Denayer et al. ${ }^{45}$ found $n$-pentane $62.1 \mathrm{~kJ} / \mathrm{mol}$ (simulation 61.96 $\mathrm{kJ} / \mathrm{mol}$ ), $n$-hexane $75.0 \mathrm{~kJ} / \mathrm{mol}$ (simulation $72.5 \mathrm{~kJ} / \mathrm{mol}$ ), $n$ heptane $87.9 \mathrm{~kJ} / \mathrm{mol}$ (simulation $83.6 \mathrm{~kJ} / \mathrm{mol}$ ), $n$-octane 100.5 $\mathrm{kJ} / \mathrm{mol}$ (simulation $95.1 \mathrm{~kJ} / \mathrm{mol}$ ).

MWW structures have two independent 10-ring pore systems, a large cavity $(0.71 \mathrm{~nm} \times 1.8 \mathrm{~nm})$ pore system, and a channeltype $(0.4 \mathrm{~nm} \times 0.55 \mathrm{~nm})$ pore system. The computed isotherms for $n$-hexane at various temperatures are shown in Figure 14. The experimental results are the data of Du et al. ${ }^{49}$ Considering the complexity of the experimental measurements the agreement is good. Much of the complexity originates from the existence of $0.9 \mathrm{~nm}$ deep pockets on the external surface that may have adsorption properties similar to that of the intra-crystalline region. This phenomenon obscures especially the lower temperature results of $\mathrm{Du}$ et al.

The heats of adsorption computed at $300 \mathrm{~K}$ are $54.0 \mathrm{~kJ} / \mathrm{mol}$
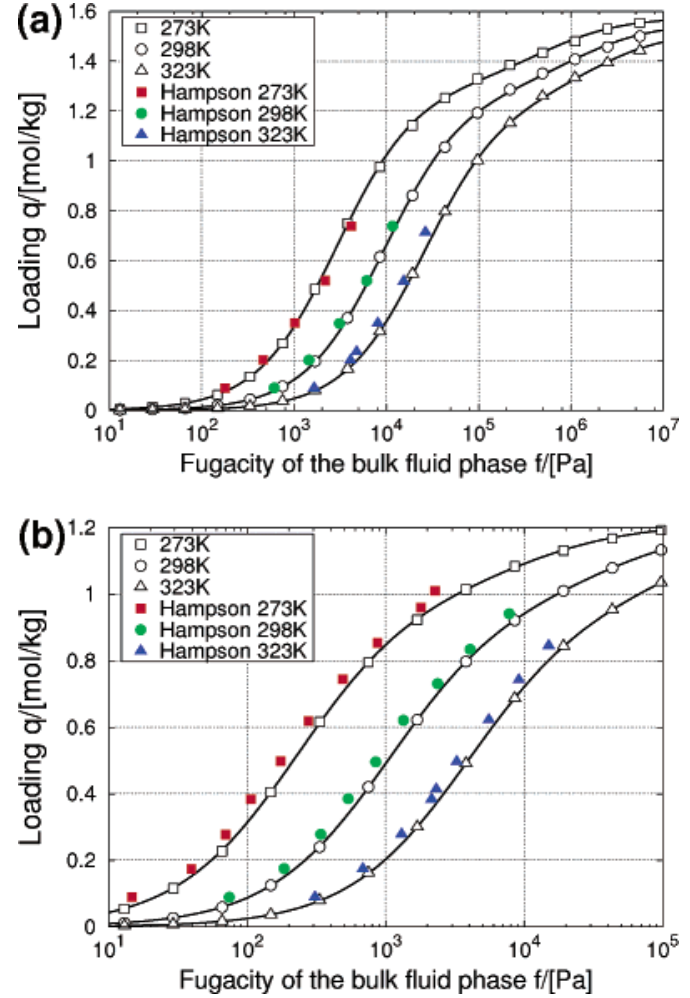

Figure 13. Isotherms of linear alkanes a) ethane and b) propane in TON at various temperatures. Experimental data are taken from Hampson and Rees. ${ }^{48}$

for $n$-hexane, $59.15 \mathrm{~kJ} / \mathrm{mol}$ for 3-methylpentane, and $59.24 \mathrm{~kJ} /$ mol for 2-methylpentane. Du et al. obtained $38.0 \mathrm{~kJ} / \mathrm{mol}$ for $n$-hexane computed from the van't Hoff plot, and $46.9 \mathrm{~kJ} / \mathrm{mol}$ from the isotherms. The former is inaccurate because the data were determined too far outside the Henry regime, and the latter is inaccurate due to intrusion by external surface adsorption at low temperature. Interestingly, the heat of adsorption is not directly proportional to the carbon number (Figure 15), because the MWW combines two effects: the linear behavior of channeltype zeolites and the nonlinear, periodic behavior of the heat of adsorption in cage/window-type systems. ${ }^{51,52}$

\section{Discussion}

Simulations are becoming increasingly less expensive, faster, and more accurate. Simulations utilizing the current force field 


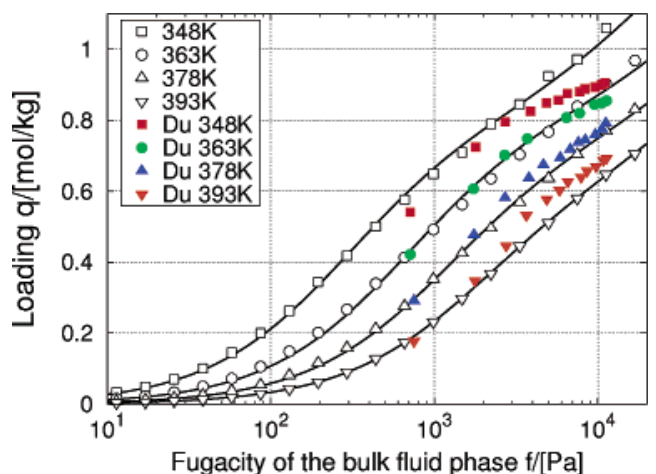

Figure 14. Isotherm of $n$-hexane in MWW at various temperatures. Experimental data is taken from $\mathrm{Du}$ et al. ${ }^{49}$

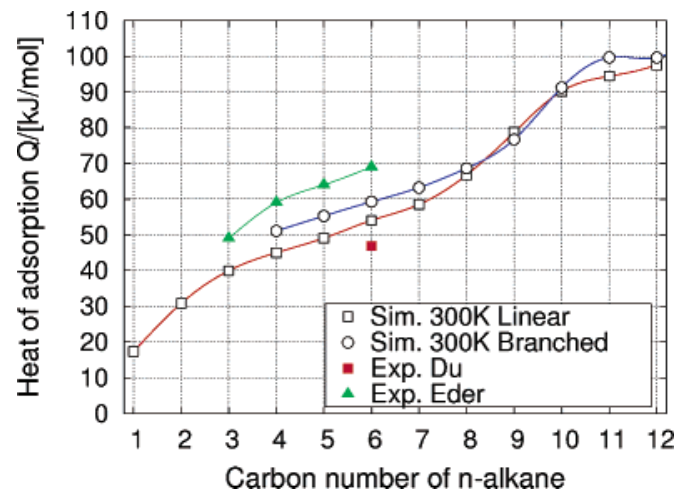

Figure 15. Heat of adsorption of linear and mono-branched alkanes as a function of carbon number in MWW, computed at $300 \mathrm{~K}$. Experimental data are taken from Du et al. ${ }^{49}$ and Eder et al. ${ }^{50}$ on a protonated MWW zeolite.

afford valuable guidance for experimental adsorption research. First, it can serve as a reference. Before doing any experiments, the model can predict the type of the isotherm, low-coverage properties such as the heats of adsorption and Henry coefficients, and the maximum loading. Interesting pressure and temperatures regimes can be identified, and the range of the Henry regime can be established. A second practical use of these simulations is to resolve experimental discrepancies. As an example, we have scrutinized the available experimental data and have highlighted the lack of low or high pressure data as a common source for error. Experimental measurements in suboptimal pressure regimes can explain the high scatter found in the Henry coefficients and heats of adsorption as reported by various groups. A third advantage of simulations is its predictive power. We predict a surprisingly nonlinear dependence of the heat of adsorption on carbon number for MWW-type zeolites, that might inspire experimentalists to verify this dependence. A fourth use of simulations is the explanation of adsorption data on a molecular level. Simulations can forge the connection between the location of the adsorbates inside the channels and cages and peculiarities (such as inflection points) in the adsorption isotherm. These explanatory data are very difficult to obtain experimentally. For adsorption of mixtures in zeolites, CBMC simulations have revealed new ways of separating linear and branched alkanes by exploiting subtle entropy effects. ${ }^{53}$

We like to comment on the application of the current model to diffusion in molecular sieves. The currently proposed model faithfully reproduces the inflection points in isotherms. Proper reproduction of the inflection is necessary, since an inflection in the isotherm leads to a sharp inflection in the diffusion behavior. ${ }^{54,55}$ The adsorbent-adsorbate parameters are uniquely determined, and in that sense, the model can be directly applied to diffusion in zeolites. However, it remains to be seen if the united atom approximation also holds for diffusion in molecular sieves. There seems to be some indication that framework vibrations can alter the diffusivities of tightly fitting molecules, ${ }^{56,57}$ even though this appears not to be the case for the diffusion of small alkanes through cation-free sieves. ${ }^{58,59} \mathrm{We}$ stress that to compare a flexible framework with a rigid framework the flexibility should be modeled in such a way that the two structures are on average identical. This implies that the reference bond lengths should be taken from the rigid structure. ${ }^{11}$

In most nanoporous framework structures, the large oxygen atoms shield the much smaller silicon, aluminum, and phosphorus atoms. Therefore, the model only needs to consider interactions between the adsorbate and the oxygen atoms, provided there is no net negative electrical charge on the framework. ${ }^{12}$ Theoretical studies have suggested that the electron density on a charge-neutral framework is lower in an aluminophosphate than in silica, which would induce a lower polarization and a lower heat of adsorption for alkanes. Some authors found experimental support for this theory, whereas others found none (see for discussion ref 60 and reference therein). If the latter are correct, this would extend the applicability of our parameters to aluminophosphates and possibly even to more recently described nanoporous framework materials based on sulfur or nitrogen instead of oxygen atoms. In principle, one can extend the force field to adsorption of alkanes in pillared clays. ${ }^{61} \mathrm{~A}$ further extension would be to include more types of pseudo atoms. Although the fitting procedure is applied to hydrocarbons, it is by no means restricted to alkanes. In the literature, many isotherms with inflections can be found, and these molecules can easily be included.

\section{Conclusions}

A united atom model is presented that is capable of a quantitative prediction of adsorption properties of both linear and branched alkanes in charge neutral molecular sieves. Very good agreement between experimental and simulated isotherms was found for AFI-, MFI-, TON-, DDR-, and MWW-type structures over a wide range of pressures and temperatures. The simulations highlight three common sources for discrepancies between experimental data sets: (1) a lack of low pressure data, (2) a lack of high pressure data, and (3) the too short experimental equilibration times. These can explain the large scatter in the experimentally reported values for the heat of adsorption and the Henry coefficients. The united atom molecular simulation results afford selection of the experimentally most sound values, and afford prediction of these values if none are available experimentally. This should be of great value when studying the use of nanoporous framework structures in industrial separation or catalytic processes and is particularly advantageous for mixtures, for which very few experimental data are available.

Acknowledgment. We thank The Netherlands Research Council for Chemical Sciences $(\mathrm{CW})$ and E. Beerdsen, P. Bolhuis, E. J. Meijer, M. Dreischor, and M. Schenk for valuable discussions, suggestions, and comments on our manuscript.

\section{Appendix}

a. Adsorption Ensemble. In adsorption studies, one would like to know the amount of materials adsorbed as a function of pressure and temperature of the reservoir with which the sieve is in contact. Therefore, the natural ensemble to use is the grand- 
canonical ensemble (or $\mu, V, T$ ensemble). In this ensemble, the temperature, $T$, the volume, $V$, and the chemical potential, $\mu$, are fixed. The equilibrium conditions are that the temperature and chemical potential of the gas inside and outside the adsorbent must be equal. The imposed chemical potential $\mu$ can be related to the fugacity $f$

$$
\beta \mu=\beta \mu_{\mathrm{id}}^{0}+\ln (\beta f)
$$

where $\beta=1 /\left(k_{\mathrm{B}} T\right)$, with $k_{\mathrm{B}}$ the Boltzmann constant, and $\mu_{\mathrm{id}}^{0}$ is the reference chemical potential. The pressure $p$ is related to the fugacity $f$ by

$$
f=\phi p
$$

where $\phi$ is the fugacity coefficient computed directly from the equation of state of the vapor in the reservoir. For all adsorbates, the experimental equation of state is well-known, and we use the Peng-Robinson equation of state to convert the pressure to the corresponding fugacity, introducing only a small correction for the currently studied systems.

b. Configurational Bias Monte Carlo (CBMC). Conventional Monte Carlo is time-consuming for long chain molecules. The fraction of successful insertions into the sieve is too low. To increase the number of successfully inserted molecules, we apply the CBMC technique. ${ }^{6,20,62}$ In the CBMC scheme, it is convenient to split the total potential energy $U$ of a trial site into two parts

$$
U=U^{\mathrm{int}}+U^{\mathrm{ext}}
$$

The first part is the internal, bonded potential $U^{\text {int }}$ which is used for the generation of trial orientations. The second part of the potential, the external potential $U^{\text {ext }}$, is used to bias the selection of a site from the set of trial sites. This bias is exactly removed by adjusting the acceptance rules. In the CBMC technique, a molecule is grown segment-by-segment. For each segment, we generate a set of $k$ trial orientations according to the internal energy $U^{\text {int }}$ and compute the external energy $U_{i}^{\text {ext }}(j)$ of each trial position $j$ of segment $i$. In this work, the number of trial positions $k$ for both NVT and $\mu \mathrm{VT}$ is set to 10 . We select one of these trial positions with a probability

$$
P_{\mathrm{i}}(j)=\frac{\mathrm{e}^{-\beta^{U_{\mathrm{i}}^{e x t}}(j)}}{\sum_{l=1}^{k} \mathrm{e}^{-\beta_{\mathrm{i}_{\mathrm{i}}^{e x t}}(l)}}=\frac{\mathrm{e}^{-\beta^{U_{\mathrm{i}}^{e x t}}(j)}}{w(i)}
$$

The selected trial orientation is added to the chain, and the procedure is repeated until the entire molecule has been grown. For this newly grown molecule, we compute the so-called Rosenbluth factor

$$
W^{\mathrm{new}}=\prod_{i} w(i)
$$

To compute the old Rosenbluth factor $W^{\text {old }}$ of an already existing chain, $k-1$ trial orientations are generated for each segment. These orientations, together with the already existing bond, form the set of $k$ trial orientations. In a dynamic scheme, a Markov chain of states is generated. The average of a property is the average of over the elements of the Markov chain. For an infinite Markov chain, the expression is exact. Every new configuration is accepted or rejected using an acceptance/rejection rule.

We have defined $\mu^{\mathrm{ex}}$ as the difference in chemical potential of the interacting alkane and an alkane in the ideal gas state.
The Rosenbluth weight $\left\langle W^{\mathrm{IG}}\right\rangle$ of the reference state of the ideal gas has to be computed in separate simulation. This quantity is needed when comparing with real experimental data.

c. Energy Computation. We describe in some detail the computation of the energies using CBMC for our molecular united atom model. The total energy $U$ is split into two contributions

$$
U=U^{\mathrm{int}}+U^{\mathrm{ext}}
$$

The internal energy $U^{\text {int }}$ is given by

$$
U^{\text {int }}=U^{\text {bond }}+U^{\text {bend }}+U^{\text {torsion }}
$$

with

$$
\begin{gathered}
U^{\text {bond }}=\sum_{\text {bonds }} \frac{1}{2} k_{1}\left(r-r_{0}\right)^{2} \\
U^{\text {bend }}=\sum_{\text {bends }} \frac{1}{2} k_{2}\left(\cos \theta-\cos \theta_{0}\right)^{2} \\
U^{\text {torsion }}=\sum_{\text {torsions }} \sum_{n=0}^{5} \eta_{n} \cos ^{n} \phi
\end{gathered}
$$

where $k_{1} / k_{\mathrm{B}}=96500 \mathrm{~K} / \AA^{2}$ is the bond energy constant, $r_{0}=$ $1.54 \AA$ the reference bond length, $k_{2} / k_{\mathrm{B}}=62500 \mathrm{~K} / \mathrm{rad}^{2}$ the bend energy constant, $\theta_{0}=114^{\circ}$ the reference bend angle, $\phi$ the dihedral angle (defined as $\phi_{\text {trans }}=0$ ), and $\eta_{n} / k_{\mathrm{B}}$ in $\mathrm{K}$ denote the six torsion parameters. The torsion potential around $\mathrm{A}-\mathrm{B}$ is not split up in several torsions. When $\mathrm{A}=\mathrm{CH}_{2}$ or $\mathrm{B}=\mathrm{CH}_{2}$, a dummy hydrogen is added to this group. The dummy atom does not have any nonbonded interactions, only bendings and a single torsion interaction. The external energy $U^{\text {ext }}$ consists of a guest-guest intermolecular energy $U^{\text {gg }}$, a host-guest interaction $U^{\text {hg }}$, and an intramolecular Lennard-Jones interaction $U^{\text {intra }}$ for beads in a chain separated by more than three bonds

$$
U^{\mathrm{ext}}=U_{i j}^{\mathrm{gg}}+U_{i j}^{\mathrm{hg}}+U_{i j}^{\mathrm{intra}}
$$

with

$$
U_{i j}^{\mathrm{gg}, \mathrm{hg}, \text { intra }}=\sum_{\mathrm{LJ}-\text { pairs }} 4 \epsilon_{i j}\left[\left(\frac{\sigma_{i j}}{r_{i j}}\right)^{12}-\left(\frac{\sigma_{i j}}{r_{i j}}\right)^{6}\right]-E_{\mathrm{cut}}
$$

where $r_{i j}$ is the distance between site $i$ and site $j, r_{\text {cut }}=12.0 \AA$, the cutoff radius, $E_{\text {cut }}$ the energy at the cutoff radius, and $U_{i j}^{\mathrm{gg}, \mathrm{hg} \text {,intra }}=0$ when $r_{i j}>r_{\text {cut. }}$. The Lennard-Jones potential consists of two parameters, $\sigma$ is the size parameter, and $\epsilon$ is the strength parameter. The force field is described by the parameters listed in Table 3.

d. Monte Carlo Moves. Several Monte Carlo moves can be employed during a simulation.

Displacement Move. A chain is selected at random and given a random displacement. The maximum displacement is taken such that $50 \%$ of the moves is accepted. The acceptance rule is

$$
\operatorname{acc}(\text { old } \rightarrow \text { new })=\min \left(1, \mathrm{e}^{-\beta\left(U^{\text {new }}-U^{\text {old }}\right)}\right)
$$

Note that the energy of the new configuration $U^{\text {new }}$ and the energy of the old configuration $U^{\text {old }}$ only differ in the external energy.

Rotation Move. A chain is selected at random and given a random rotation. The center of the rotation is the center of mass. 
TABLE 6: Adsorption Properties Computed at the Infinite Dilution from a NVT Simulation ${ }^{a}$

\begin{tabular}{lll}
\hline \multicolumn{1}{c}{ property } & \multicolumn{1}{c}{ formula } & $\mathrm{units}$ \\
\hline Henry coefficient $K_{\mathrm{H}}$ & $K_{\mathrm{H}}=\left(1 / R T \rho_{f}\right)\langle W\rangle /\left\langle W^{\mathrm{IG}}\right\rangle$ & $\mathrm{mol} / \mathrm{kg} / \mathrm{Pa}$ \\
internal energy $\Delta U$ & $\Delta U=\left\langle U_{\mathrm{hg}}-\left\langle U_{\mathrm{h}}\right\rangle-\left\langle U_{\mathrm{g}}\right\rangle\right.$ & $\mathrm{J} / \mathrm{mol}$ \\
Helmholtz free energy $\Delta A$ & $\Delta A=-R T \ln \left(\langle W\rangle /\left\langle W^{\mathrm{IG}}\right\rangle\right)$ & $\mathrm{J} / \mathrm{mol}$ \\
Gibbs free energy $\Delta G$ & $\Delta G=\Delta A-R T$ & $\mathrm{~J} / \mathrm{mol}$ \\
isosteric enthalpy of adsorption $\Delta H$ & $\Delta H=-\partial \ln \left(K_{\mathrm{H}}\right) / \partial(R T)^{-1}=\Delta U-R T$ & $\mathrm{~J} / \mathrm{mol}$ \\
isosteric heat of adsorption $Q$ & $Q=-\Delta H$ & $\mathrm{~J} / \mathrm{mol}$ \\
entropy $\Delta S$ & $\Delta S=(\Delta U-\Delta A) / T=(\Delta H-\Delta G) / T$ & $\mathrm{~J} /(\mathrm{mol} \mathrm{K})$
\end{tabular}

${ }^{a}$ The Rosenbluth factor $\langle W\rangle$, the Rosenbluth factor of an ideal chain $\left\langle W^{1 G}\right\rangle$, the ensemble average of the potential energy of the host-guest system $\left\langle U_{\mathrm{hg}}\right\rangle$, the energy of an isolated ideal chain $\left\langle U_{\mathrm{g}}\right\rangle$, and the average host energy $\left\langle U_{\mathrm{h}}\right\rangle$ (zero for a rigid framework) are computed from two independent simulations of a single chain: a NVT-simulation of a chain adsorbed in the framework and a NVT simulation of an isolated chain in the ideal gas phase. Here, $T$ is the temperature, $R=8.31451 \mathrm{~J} /(\mathrm{mol} \mathrm{K})$ the gas constant, and $\rho_{\mathrm{f}}$ in $\mathrm{kg} / \mathrm{m}^{3}$ the density of the framework.

The maximum rotation angle is selected such that $50 \%$ of the moves are accepted. The acceptance rule is given by eq 15 . Again, the energy of the new configuration $U^{\text {new }}$ and the energy of the old configuration $U^{\text {old }}$ only differ in the external energy.

Insertion Move. A chain is grown at a random position. The acceptance rule for insertion of the particle is given by

$$
\operatorname{acc}(N \rightarrow N+1)=\min \left(1, \frac{W^{\text {new }} \beta V}{N+1} \frac{f}{\left\langle W^{\mathrm{IG}}\right\rangle}\right)
$$

Deletion Move. A chain is chosen at random and the old Rosenbluth factor is computed. The acceptance rule for deletion of the particle is given by

$$
\operatorname{acc}(N \rightarrow N-1)=\min \left(1, \frac{N}{W^{\text {old }} \beta V} \frac{\left\langle W^{\mathrm{IG}}\right\rangle}{f}\right)
$$

Full Regrow Move. A chain is selected at random and is completely regrown at a random position. This move is essential for $N V T$ to change the internal configuration of a molecule, and during this move, data for the average Rosenbluth weight can be collected. The acceptance rule for full regrow is given by

$$
\operatorname{acc}(\text { old } \rightarrow \text { new })=\min \left(1, \frac{W^{\text {new }}}{W^{\text {old }}}\right)
$$

Partial Regrow Move. A chain is selected at random and part of the molecule is regrown. It is decided at random which part of the chain is regrown and with which segment the regrown is started. The acceptance rule for partial regrow is given by eq 18.

Identity Change Move (Mixtures). The identity-change trial move ${ }^{63}$ is called semi-grand ensemble, but it can also be seen as a special case of the Gibbs ensemble. One of the components is selected at random and an attempt is made to change its identity. The acceptance rule is given by ${ }^{64}$

$$
\operatorname{acc}(A \rightarrow B)=\min \left(1, \frac{W^{\text {new }} f_{\mathrm{B}} N_{\mathrm{A}}}{W^{\text {old }} f_{\mathrm{A}}\left(N_{\mathrm{B}}+1\right)}\right)
$$

where $f_{\mathrm{A}}$ and $f_{\mathrm{B}}$ are the fugacities of components $A$ and $B$, and $N_{\mathrm{A}}$ and $N_{\mathrm{B}}$ are the number of particles.

The relative probabilities for attempting these moves were such that in the NVT-simulations $10 \%$ of the total number of moves were displacements, $10 \%$ rotations, $10 \%$ partial regrowths, and $70 \%$ regrowths of the entire molecule. For the case of grand-canonical simulations of the pure components the distribution of moves was: $15 \%$ displacements, $15 \%$ rotations, $15 \%$ partial regrowths, and $55 \%$ exchanges with the reservoir. For alkane mixtures the number of exchanges was reduced to
$50 \%$ and the remaining $5 \%$ of the moves were attempts to change the identity of a molecule.

e. Duration/Length of Simulation. Simulations are performed in cycles. The number of cycles needed for equilibration depends on the number of molecules. We define a cycle to consists of smaller steps proportional to the number of molecules with 20 as the minimum

$$
N_{\text {cycles }}=\max (20, N) \times N_{\text {steps }}
$$

In each step one Monte Carlo move is performed. For molecules smaller than pentane, at least $5 \times 10^{5}$ cycles are used to compute the isotherms. For longer molecules and all NVT simulations, we used at least $1 \times 10^{6}$ cycles.

f. Computation of Low-Coverage Adsorption Properties. If the chemical potential is sufficiently low, the loading $q$ is proportional to the Henry coefficient $K_{\mathrm{H}}$ and the pressure

$$
q=K_{\mathrm{H}} p
$$

The Henry coefficient is related to the Rosenbluth factor

$$
K_{\mathrm{H}}=\frac{1}{R T \rho_{\mathrm{f}}} \frac{\langle W\rangle}{\left\langle W^{\mathrm{IG}}\right\rangle}
$$

where $\rho_{\mathrm{f}}$ is the density of the framework. The chemical potential is related to the Helmholtz free energy $A$

$$
\mu=\left(\frac{\partial A}{\partial N}\right)_{V, T}
$$

In the infinite dilution limit

$$
\Delta A=A(1)-A(0)=\mu
$$

Therefore the Helmholtz free energy can be computed from a NVT simulation

$$
\Delta A=-R T \ln \frac{\langle W\rangle}{\left\langle W^{\mathrm{IG}}\right\rangle}
$$

The entropy $\Delta S$ is given by

$$
\Delta S=\frac{(\Delta U-\Delta A)}{T}
$$

or equivalently

$$
\Delta S=\frac{(\Delta H-\Delta G)}{T}
$$

In the limit of zero coverage, the Henry coefficient is related to the enthalpy of adsorption at a fixed loading $\Delta H$ via a thermodynamic relation 


$$
\Delta H=-\frac{\partial \ln \left(K_{\mathrm{H}}\right)}{\partial(R T)^{-1}}
$$

In a simulation, the isosteric enthalpy can be computed more conveniently from the internal energy difference

$$
\Delta H=\Delta U-R T
$$

From eqs 26, 27, and 29, we obtain for the Gibbs free energy difference $\Delta G$ at infinite dilution

$$
\Delta G=\Delta A-R T
$$

The formulas are summarized in Table 6 .

\section{References and Notes}

(1) Blauwhoff, P. M. M.; Gosselink, J. W.; Kieffer, E. P.; Sie, S. T.; Stork, W. H. J. In Catalysis and Zeolites; Weitkamp, J., Puppe, L., Eds.; Springer: Berlin, 1999; pp 437-538. 118.

(2) Thomas, J. M. Solid Acid Catalysis. Sci. Am. 1992, 266, 112-

(3) Haag, W. O. In Zeolites and Related Microporous Materials: State of the Art 1994; Studies in Surface Science and Catalysis; Weitkamp, J., Karge, H. G., Pfeifer, H., Hölderich, W., Eds.; Elsevier: Amsterdam, 1994; Vol. 84, pp 1375-1394.

(4) Rozanska, X.; van Santen, R. A.; Hutschka, F. S. Prog. Theor. Chem. Phys. 2001, 1-28.

(5) Macedonia, M. D.; Maginn, E. J. Mol. Phys. 1999, 96, 1375-1390.

(6) Smit, B.; Siepmann, J. I. J. Phys. Chem. 1994, 98, 8442-8452.

(7) Vlugt, T. J. H.; Krishna, R.; Smit, B. J. Phys. Chem. B 1999, 103, $1102-1118$.

(8) Lachet, V.; Boutin, A.; Pellenq, R. J.-M.; Nicholson, D.; Fuchs, A. H. J. Phys. Chem. 1996, 100, 9006-9013.

(9) Clark, L. A.; Sierka, M.; Sauer, J. J. Am. Chem. Soc. 2003, 125 , 2136-2141.

(10) Ryckaert, J. P.; Bellemans, A. Faraday Dicuss. Chem. Soc. 1978, $66,95-106$.

(11) Vlugt, T. J. H.; Schenk, M. J. Phys. Chem. B 2002, 106, 1275712763

(12) Bezus, A. G.; Kiselev, A. V.; Lopatkin, A. A.; Du, P. Q. J. J. Chem. Soc., Faraday Trans. 2 1978, 74, 367-379.

(13) van Koningsveld, H.; van Bekkum, H.; Jansen, J. C. Acta Crystallogr. 1987, B43, 127-132.

(14) Marler, B. Zeolites 1987, 7, 393-397.

(15) Qiu, S.; Pang, W.; Kessler, H.; Guth, J. L. Zeolites 1989, 9, 440444.

(16) Gies, H. Z. Kristallogr. 1986, 175, 93-104.

(17) Camblor, M. A.; Corma, A.; Diaz-Cabanas, M.-J.; Baerlocher, Ch.

J. Phys. Chem. B 1998, 102, 44-51.

(18) Smit, B.; Maesen, T. L. M. Nature 1995, 374, 42-44.

(19) Smit, B.; Siepmann, J. I. J. Phys. Chem. 1996, 98, 8442-8452.

(20) Frenkel, D.; Smit, B. Understanding molecular simulation, 2nd ed.; Academic Press: London, 2002.

(21) Martin, C.; Tosi-Pellenq, N.; Patarin, J.; Coulomb, J. P. Langmuir 1998, 14, 1774-1778.

(22) Smit, B. J. Phys. Chem. 1995, 99, 5597-5603.

(23) June, R. L.; Bell, A. T.; Theodorou, D. N. J. Phys. Chem. 1992, 96, $1051-1060$

(24) Pascual, P.; Ungerer, P.; Tavitian, B.; Pernot, P.; Boutin, A. Phys. Chem. Chem. Phys. 2003, 5, 3684-3693.

(25) Smit, B.; Loyens, L. D. J. C.; Verbist, G. L. M. M. Faraday Discuss. 1997, 106, 93-104.

(26) Du, Z.; Vlugt, T. J. H.; Smit, B.; Manos, G. AIChE J. 1998, 44, $1756-1764$.

(27) Sun, M. S.; Talu, O.; Shah, D. B. J. Phys. Chem. 1996, 100, 1727617280
(28) Vlugt, T. J. H.; Zhu, W.; Kapteijn, F.; Moulijn, J. A.; Smit, B.; Krishna, R. J. Am. Chem. Soc. 1998, 120, 5599-5600.

(29) Cavalcante, C. L.; Ruthven, D. M. Ind. Eng. Chem. Res. 1995, 34, $177-184$.

(30) Jolimaitre, E.; Tayakout-Fayolle, M.; Jallut, C.; Ragil, K. Ind. Eng. Chem. Res. 2001, 40, 914-926.

(31) Jolimaitre, E.; Ragil, K.; Tayakout-Fayolle, M.; Jallut, C. AIChE J. 2002, 48, 914-926.

(32) Eder, L. Thermodynamic Siting of Alkane Adsorption in Molecular Sieves. Ph.D. Thesis, 1996.

(33) Zhu, W.; Kapteijn, F.; Moulijn, J. A. Phys. Chem. Chem. Phys. 2000, 2, 1989-1995.

(34) Zhu, W.; Kapteijn, F.; van der Linden, B.; Moulijn, J. A. Phys. Chem. Chem. Phys. 2001, 3, 1755-1761.

(35) Zhu, W.; Kapteijn, F.; Moulijn, J. A. Adsorption 2000, 6, 159167.

(36) Sun, M. S.; Shah, D. B.; Talu, O. J. Phys. Chem. B 1998, 102, $1466-1473$

(37) Choudhary, V. R.; Mayadevi, S. Zeolites 1996, 17, 501-507.

(38) Yang, Y.; Rees, L. V. C. Microporous Mater. 1997, 12, 223-228.

(39) van Well, W. J. M.; Wolthuizen, J. P.; Smit, B.; van Hoof, J. H.

C.; van Santen, R. A. Angew. Chem., Int. Ed. Engl. 1995, 34, 2543-2544.

(40) Meyer, K. M. A. De; Chempath, S.; Denayer, J. F. M.; Martens, J.

A.; Snurr, R. Q.; Baron, G. V. J. Phys. Chem. B 2003, 107, 10760-10766.

(41) Martin, M. G.; Siepmann, J. I. J. Phys. Chem. B 1999, 103, 45084517.

(42) Schuring, D.; Koriabkina, A. O.; de Jong, A. M.; Smit, B.; van Santen, R. A. J. Phys. Chem. B 2001, 105, 7690-7698.

(43) Krishna, R.; Calero, S.; Smit, B. Chem. Eng. J. 2002, 88, 81-94.

(44) Arik, I. C.; Denayer, J. F.; Baron, G. V. Microporous Mesoporous Mater. 2003, 60, 111-114.

(45) Denayer, J. F.; Souverijns, W.; Jacobs, P. A.; Martens, J. A.; Baron,

G. V. J. Phys. Chem. B 1998, 102, 4588-4597.

(46) Zhu, W.; Kapteijn, F.; Moulijn, J. A.; den Exter, M. C.; Jansen, J. C. Langmuir 2000, 16, 3322-3329.

(47) Zhu, W.; Kapteijn, F.; Moulijn, J. A.; Jansen, J. C. Phys. Chem Chem. Phys. 2000, 2, 1773-1779.

(48) Hampson, J. A.; Rees, L. V. C. Stud. Surf. Sci. Catal. 1994, 83, 197-208.

(49) Du, H.; Kalyanaraman, M.; Camblor, M. A.; Olsen, D. H Microporous Mesoporous Mater. 2000, 40, 305-312.

(50) Eder, F.; Lercher, J. A. J. Phys. Chem. B 1997, 101, 1273.

(51) Dubbeldam, D.; Calero, S.; Maesen, T. L. M.; Smit, B. Phys. Rev Lett. 2003, 90, 245901.

(52) Dubbeldam, D.; Calero, S.; Maesen, T. L. M.; Smit, B. Angew. Chem., Int. Ed. 2003, 42, 3624-3626.

(53) Krishna, R.; Smit, B.; Calero, S. Chem. Soc. Rev. 2002, 31, 185194.

(54) Krishna, R.; Paschek, D. Chem. Eng. J. 2002, 85, 7-15.

(55) Krishna, R.; Vlugt, T. J. H.; Smit, B. Chem. Eng. Sci. 1999, 54, $1751-1757$

(56) Sastre, G.; Catlow, C. R. A.; Corma, A. J. Phys. Chem. B 1999, $103,5187$.

(57) Bouyermaouen, A.; Bellemans, A. J. Chem. Phys 1998, 108, 2170.

(58) Demontis, P.; Suffritti, G. B.; Fois, E. S.; Quartieri, S. J. Phys. Chem. 1992, 96, 1482-1490.

(59) Fritzche, S.; Wolfsberg, M.; Haberlandt, R.; Demontis, P.; Suffritti,

G. B.; Tilocca, A. Chem. Phys. Lett. 1998, 296, 253.

(60) Eder, F.; Lercher, J. A. J. Phys. Chem. 1996, 100, 16460-16462.

(61) Cao, D.; Wang, W.; Duan, X. J. Colloid Interface Sci. 2002, 254, $1-7$.

(62) Smit, B. Mol. Phys. 1995, 85, 153-172.

(63) Martin, M. G.; Siepmann, J. I. J. Am. Chem. Soc. 1997, 119, 89218924.

(64) Panagiotopoulos, A. Z. Int. J. Thermophys. 1989, 10, 447-457. (65) Maris, T.; Vlugt, T. J. H.; Smit, B. J. Phys. Chem. B 1998, 102, $7183-7189$

(66) Martin, M. G.; Thompson, A. P.; Nenoff, T. M. J. Chem. Phys. 2001, 114, 7174-7181.

(67) Nath, S. K.; Escobedo, F. A.; de Pablo, J. J. J. Chem. Phys. 1998, $108,9905-9911$.

(68) Vlugt, T. J. H.; Frash, M. personal communication. 